\title{
Asymptotic behaviour of stationary distributions for countable Markov chains, with some applications
}

\author{
SANJAR ASPANDIIAROV ${ }^{1}$ and ROUDOLF IASNOGORODSKI ${ }^{2}$ \\ ${ }^{1}$ Université Paris V, UFR de Mathématiques et Informatique, 45 rue des Saints-Pères, 75270 \\ Paris Cedex 06, France. E-mail: aspandij@descartes.math-info.univ-paris5.fr \\ ${ }^{2}$ Université d'Orléans, MAPMO 1803, UFR Sciences BP 6759, 45067 Orléans Cedex 02, France. \\ E-mail:iasno@galois.labomath.univ-orleans.fr
}

Let $\left\{Z_{n}, n \geqslant 0\right\}$ be an aperiodic irreducible recurrent (not necessarily positive recurrent) Markov chain taking values on a countable unbounded subset $S$ of $\mathbb{R}^{d}, \pi(\cdot)$ its invariant measure and $f$ is a non-negative function defined on $S$. We first find sufficient conditions under which $\int_{S} f(z) \pi(\mathrm{d} z)=\infty$ (the corresponding result for the finiteness of $\int_{S} f(z) \pi(\mathrm{d} z)$ was obtained by Tweedie). Then we obtain lower and upper bounds for the values of the invariant measure $\pi$ on the subsets $B$ of $S$, that is, $\pi(B)$. These bounds are expressed in terms of first passage probabilities and the first exit time from $B$. We also show how to estimate the latter quantities using sub- or supermartingale techniques. The results are finally illustrated for driftless reflected random walks in $\mathbb{Z}_{+}^{2}$ and for Markov chains on nonnegative reals with asymptotically small drift of Lamperti type. In both cases we obtain very precise information on the asymptotic behaviour of their stationary measures.

Keywords: occupation time; recurrent Markov chain; reflected random walk; stationary measure; submartingale; supermartingale

\section{Introduction}

The main object of this paper is the study of the asymptotic behaviour of the stationary distributions for recurrent Markov chains. This question appears as a natural development of the classical problem of existence and uniqueness of stationary measures and is known to be important for the ergodic theory of Markov chains. In the particular situation of Markov chains satisfying the condition of so-called geometric ergodicity, the characterization of the invariant measure is well understood - for further information, see Fayolle et al. (1994), Nummelin and Tweedie (1978; 1994), Nummelin and Tuominnen (1982), Meyn and Tweedie (1993) and references therein. However, as far as we know, even in the case of subgeometric ergodicity, there are only few results providing similar information. One of the first was obtained by Tweedie (1983), who found sufficient conditions under which the stationary distribution $\pi$ admits moments of the general form $\int f(x) \pi(\mathrm{d} x)$ for non-negative functions $f$. We have recently learned that Menshikov and Popov (1995) have proved some results concerning the values of $\pi$ on some subsets $B$ of the state space for subgeometric positive 
recurrent Markov chains with bounded increments based on relations between first passage times and stationary probabilities. More difficult appears to be the case of null recurrent Markov chains when first passage times and the first return times have infinite expectations and the stationary probabilities cannot be expressed in terms of the first return times.

Our principal goal is to propose a unified approach that enables one to obtain a description of the stationary measure $\pi$ of recurrent (not necessarily positive recurrent) countable Markov chains. The main results are proved in Section 2. Here we first complete Tweedie's result by obtaining sufficient conditions for divergence of $\int f(x) \pi(\mathrm{d} x)$ for nonnegative functions $f$ (Theorem $1^{\prime}$ ). In passing, we note that when $f$ is bounded by some positive constant $c$ these conditions imply the null recurrence of a Markov chain. Next we give upper and lower bounds for $\pi(B)$ in terms of first passage probabilities and the first exit time from the set $B$ (Theorems 2 and 3 ). We do this by directly counting excursions of the Markov chain hitting $B$ outside a fixed finite set $A$ which in turn enables us to estimate the total occupation time of $B$ between two successive visits of $A$. We finally show how to estimate the quantities appearing in these bounds by means of sub- or supermartingale techniques (Lemmas 1 and 2).

Then, in Sections 3-4, we illustrate the results obtained on two classes of Markov chains. The first is driftless reflected random walks in a quadrant, studied recently in Fayolle et al. (1992), Aspandiiarov et al. (1996) and Aspandiiarov and Iasnogorodski (1997; 1998). The other class is countable Markov chains on non-negative real numbers with asymptotically small drift of Lamperti type, introduced in Lamperti (1963). In both cases applying the results of the first part, and constructing appropriate sub- or supermartingales, we obtain very sharp conditions of integrability (non-integrability) of functions with respect to the stationary measures, as well as bounds on the 'tails' of these measures (Theorems 7-10 and 4-5). Moreover, we are able to distinguish the asymptotic behaviour of the invariant measure on the boundary and in the interior of the quadrant. These results turn out to be very important for the recurrent classification of the three-dimensional driftless reflected random walks. Finally, it should be said that the results for reflected random walks are in concordance with corresponding results for reflected Brownian motion in a wedge obtained by Williams (1985), which is not surprising bearing in mind one recent result in Aspandiiarov (1994) on the convergence of reflected random walks to a Brownian motion. Moreover, the results obtained go beyond the Brownian motion case covering the situation when the Brownian motion is absorbed at the origin.

\section{General results on stationary distributions for countable recurrent Markov chains}

Let $(\Omega, \mathscr{F}, P)$ be a probability space with a filtration $\left\{\mathscr{F}_{n}\right\}_{n \geqslant 0}$. Throughout this section, $\left\{Z_{n}, n \geqslant 0\right\}$ is a discrete-time $\left\{\mathscr{F}_{n}\right\}$-adapted irreducible aperiodic Markov chain taking values in an unbounded countable subset $S$ of $\mathbb{R}^{d}$. We assume that the chain is recurrent with unique (up to a multiplicative constant) stationary measure $\pi$ (see Chung 1967). 


\subsection{Integrability/non-integrability of functions with respect to stationary distribution}

One of first questions regarding the measure $\pi$ to be asked is the finiteness of

$$
\mathrm{E}_{\pi} f=\sum_{z \in S} f(z) \pi(z)\left(\equiv \int f(z) \pi(\mathrm{d} z)\right),
$$

for a non-negative function $f$ defined on $S$. An answer to this question is provided by Tweedie, which we reproduce here in a slightly different form.

Definition 1. For any subset $A$ of $S$ and any initial state $Z_{0}=z$, we will denote by $\tau_{A, z}$ the first passage time into $A: \tau_{A, z}=\inf \left\{n \geqslant 1 ; Z_{n} \in A\right\}$. Notice that since the chain is recurrent, $\tau_{A, z}$ is finite with probability 1 . We will omit the superscript $z$ when this will not cause any notational confusion.

Theorem 1 (Tweedie 1983, Theorem 1). Let $f$ be a non-negative function defined on $S$. In order that

$$
\mathrm{E}_{\pi} f<\infty,
$$

it is sufficient that for some set $A$ and some function $g$ such that $g(u) \geqslant f(u)$ on $A^{c}$, we have that for any $n \geqslant 1$ and $z \in A$, for all $z \in A, \mathrm{E}_{z}\left(g\left(Z_{1}\right) 1_{\left(\tau_{A}>1\right)}\right)$ is finite and

$$
\mathrm{E}\left(g\left(Z_{n+1}\right) 1_{\left(\tau_{A}>n+1\right)} \mid \mathscr{F}_{n}\right) \leqslant g\left(Z_{n}\right)-f\left(Z_{n}\right) P_{z} \text {-a.s. } \quad \text { on }\left\{\tau_{A}>n\right\} .
$$

A slight modification of the conditions of Theorem 1 provides the following conditions for divergence of $\mathrm{E}_{\pi} f$.

Theorem 1'. Let $f$ be a non-negative function defined on $S$. In order that

$$
\mathrm{E}_{\pi} f=\infty,
$$

it suffices that for some finite set $A$, some $z \in A$ and some function $g$ such that $\lim \sup _{n \rightarrow \infty} \mathrm{E}_{z}\left(g\left(Z_{n}\right) 1_{\left(\tau_{A}>n\right)}\right)=\infty$ and $\mathrm{E}_{z}\left(g\left(Z_{1}\right) 1_{\left(\tau_{A}>1\right)}\right)$ is finite, we have, for any $n \geqslant 1$,

$$
\mathrm{E}\left(g\left(Z_{n+1}\right) 1_{\left(\tau_{A}>n+1\right)} \mid \mathscr{F}_{n}\right) \leqslant g\left(Z_{n}\right)+f\left(Z_{n}\right) P_{z} \text {-a.s. } \quad \text { on }\left\{\tau_{A}>n\right\} .
$$

Remark 1. We need another convention. In what follows we will omit the hypothesis 'defined on $S$ ' and 'subset $A$ of $S$ ', ' $\in A \cap S$ ', implicitly assuming that all sets under study are assumed to be subsets of the state set $S$, and all functions are defined only on $S$.

Proof of Theorems 1 and 1'. The proof is easy and is based on the following well-known fact from the theory of Markov chains (see, for example, Tweedie 1983): for any $B \subseteq A^{c}$,

$$
\pi(B)=\mathrm{E}_{\pi}\left(1_{\left(Z_{0} \in A\right)} \mathrm{E}_{Z_{0}}\left(\sigma_{B}^{A}\right)\right)=\mathrm{E}_{\pi}\left(1_{\left(Z_{0} \in A\right)} \mathrm{E}_{Z_{0}}\left(\sum_{n=1}^{\infty} 1_{\left(Z_{n \wedge \tau_{A} \in B}\right)}\right)\right),
$$


where $\sigma_{B}^{A}$ is the occupation time of $B$ before the first passage into $A$, that is,

$$
\sigma_{B}^{A}=\left\{\# n ; Z_{n} \in B, \tau_{A}>n\right\} .
$$

It is then immediate that for any finite set $A$,

$$
\mathrm{E}_{\pi} f<\infty \Leftrightarrow \forall z \in A, \mathrm{E}_{z}\left(\sum_{n=1}^{\infty} f\left(Z_{n \wedge \tau_{A}}\right) 1_{\left(\tau_{A}>n\right)}\right)<\infty .
$$

Finally, iterating (1)-(2) and using the last observation, we immediately see that the desired conclusions follow from other conditions of Theorems 1 and $1^{\prime}$.

Remark 2. Notice that if $f$ is bounded from below (above) by some positive constant $\mathrm{c}$, then Theorem 1 (Theorem 1') gives sufficient conditions for positive recurrence (null recurrence) of the Markov chain $\left\{Z_{n}, n \geqslant 0\right\}$. In the positive recurrent case we simply obtain the wellknown Foster theorem (see Foster 1953).

Remark 3. Obviously, condition (1) (condition (2)) is satisfied, if whenever $z \in A^{c}$ we have, for any $n \geqslant 0$,

$$
\mathrm{E}\left(g\left(Z_{n+1}\right)-g\left(Z_{n}\right) \mid \mathscr{F}_{n}\right) \leqslant-f\left(Z_{n}\right)\left(\leqslant f\left(Z_{n}\right)\right) P_{z} \text {-a.s. } \quad \text { on }\left\{\tau_{A}>n\right\} .
$$

Remark 4. In applications the first condition on $g$ in the Theorem $1^{\prime}$ can be verified by virtue of the following observation. Suppose:

1. There is a non-negative function $h$ such that $(g(z)) /(h(z)) \rightarrow \infty$ as $|z| \rightarrow \infty$ and $|z| \rightarrow \infty$ whenever $h(z) \rightarrow \infty$.

2. Whenever $\mathrm{z}^{\prime}$ belongs to some subset $\mathscr{Z}$ of $A^{c}$, the process $\left\{h\left(Z_{n \wedge \tau_{A}}\right), n \geqslant 0\right\}$ is a $P_{z^{\prime}}$ submartingale.

3. $\mathrm{E}_{z}\left(g\left(Z_{n}\right) 1_{\left(\tau_{A}>n\right)}\right)$ is finite for any $n \geqslant 1$.

Then, whenever $z \in A$ satisfies ${ }_{A} P_{z, z^{\prime}}^{n_{0}}>0$ for some $z^{\prime} \in \mathscr{Z}$ and $n_{0}>0$, such that $h\left(z^{\prime}\right)>\sup _{x \in A} h(x)$, we have $\limsup _{n \rightarrow \infty} \mathrm{E}_{z}\left(g\left(Z_{n}\right) 1_{\tau_{A}>n}\right)=\infty\left({ }_{A} P_{z, z^{\prime}}^{n_{0}}\right.$ here is a usual $n_{0^{-}}$ step transition probability from $z$ to $z^{\prime}$ of $\left\{Z_{n}, n \geqslant 0\right\}$ with taboo set $A$ ).

Proof. Let $z, z^{\prime} \in \mathscr{Z}$ and $n_{0}>0$, satisfy ${ }_{A} P_{z, z^{\prime}}^{n_{0}}>0$ and $h\left(z^{\prime}\right)>\sup _{x \in A} h(x)$. The strong Markov property at $\tau_{\left\{z^{\prime}\right\}}$ shows that in order to prove the divergence of $\mathrm{E}_{z^{\prime}}\left(g\left(Z_{n}\right) 1_{\tau_{A}>n}\right)$ as $n \rightarrow \infty$ it suffices to verify that the family $\left\{\mathrm{E}_{z^{\prime}}\left(g\left(Z_{n \wedge \tau_{A}}\right)\right), n \geqslant 0\right\}$ is not uniformly bounded. Suppose the former assertion concerning the uniform boundedness is false. Then, using our assumptions on $h$ we would get that the family $\left\{h\left(Z_{n \wedge \tau_{A}}\right), n \geqslant 0\right\}$ is uniformly integrable and consequently $\lim _{n \rightarrow \infty} \mathrm{E}_{z^{\prime}} h\left(Z_{n \wedge \tau_{A}}\right)=\mathrm{E}_{z} h\left(Z_{\tau_{A}}\right)$. But this, with the choice of $z^{\prime}, h\left(z^{\prime}\right)>$ $\sup _{x \in A} h(x)$, contradicts

$$
\mathrm{E}_{z^{\prime}}\left(h\left(Z_{n \wedge \tau_{A}}\right)\right) \geqslant h\left(z^{\prime}\right), \quad \forall n \geqslant 0
$$




\subsection{Upper bounds for stationary distributions}

The martingale ideas of the proofs of Theorems 1 and $1^{\prime}$ can be developed further, providing us with information on $\pi(B)$ for some sets $B$.

Definition 2. Let $\partial A=\left\{z \in A ; P_{z}\left(Z_{1} \in A^{c}\right)>0\right\}$. The set $\partial A$ is non-empty since $\left\{Z_{n}, n \geqslant 0\right\}$ is an irreducible Markov chain.

Proposition 1. Under the conditions of Theorem 1 for any subset $B$ of $A^{c}$,

$$
\pi(B) \leqslant \frac{\pi(\partial A) \sup _{z \in \partial A} \mathrm{E}_{z}\left(g\left(Z_{1}\right) 1_{\left(\tau_{A}>1\right)}\right)}{\inf _{z^{\prime} \in B} f\left(z^{\prime}\right)},
$$

provided $f$ is positive on $A^{c}$.

Proof. For any $z \in \partial A$,

$$
P_{z}\left(Z_{n \wedge \tau_{A}} \in B\right)=\mathrm{E}_{z}\left(1_{\left(Z_{n} \in B\right)} 1_{\left(\tau_{A}>n\right)}\right) \leqslant \frac{\mathrm{E}_{z}\left(f\left(Z_{n}\right) 1_{\left(\tau_{A}>n\right)}\right)}{\inf _{z^{\prime} \in B} f\left(z^{\prime}\right)} .
$$

Substituting this into (3) and iterating (1)

$$
\pi(B) \leqslant \frac{\pi(\partial A) \sup _{z \in \partial A}\left(-\lim _{n \rightarrow \infty} \mathrm{E}_{z}\left(g\left(Z_{n}\right) 1_{\left(\tau_{A}>n\right)}\right)+\mathrm{E}_{z}\left(g\left(Z_{1}\right) 1_{\left(\tau_{A}>1\right)}\right)\right)}{\inf _{z^{\prime} \in B} f\left(z^{\prime}\right)} .
$$

The proof is concluded by recalling that the function $g(z)$ is non-negative on $A^{c}$.

Definition 3. Let $A, B$ be non-intersecting sets. Set $v_{B}=\tau_{B^{c}}$ and define the sets

$$
\begin{aligned}
& \Gamma_{A, B}=\left\{z \in S \backslash(A \cup B) ; P_{z}\left(\tau_{A}<\tau_{B}\right)>0 \text { and } \exists z^{\prime} \in B \text { such that } P_{z^{\prime}}\left(Z_{v_{B}}=z\right)>0\right\} ; \\
& \Gamma_{A, B}^{\prime}=\left\{z \in S \backslash(A \cup B) ; P_{z}\left(\tau_{A}<\tau_{B}\right)=0\right\} ; \\
& \Gamma_{A, B}^{\prime \prime}=\Gamma_{A, B}^{\prime} \cup B .
\end{aligned}
$$

Remark 5. Notice that by this construction the trajectory starting in $\Gamma_{A, B}^{\prime}$ cannot hit either $A$ or $\Gamma_{A, B}$ before hitting $B$. Similarly, if for any $z \in B, P_{z}\left(\tau_{A}=1\right)=0$, then the trajectory starting in $\Gamma_{A, B}^{\prime \prime}$ cannot hit $A$ before hitting $\Gamma_{A, B}$.

The principal result of this section gives an upper bound for $\pi(B)$ expressed in terms of the first passage probabilities $P_{z}\left(\tau_{\Gamma_{A, B}^{\prime \prime}}<(>) \tau_{A}\right)$, and the first exit time $v_{B}$ from $B$.

Theorem 2. Let $A$ be a finite subset of $S$ and $B$ be a subset of $A^{c}$ such that $\Gamma_{A, B}$ is non-empty and for any $z \in B, P_{z}\left(\tau_{A}=1\right)=0$. Then

$$
\pi(B) \leqslant \frac{\pi(\partial A) \sup _{z \in \partial A} P_{z}\left(\tau_{\Gamma_{A, B}^{\prime \prime}}<\tau_{A}\right) \sup _{z \in B} \mathrm{E}_{z}\left(v_{B}\right)}{\inf _{z \in \Gamma_{A, B}} P_{z}\left(\tau_{A}<\tau_{\Gamma_{A, B}^{\prime \prime}}\right) \inf _{z \in B} P_{z}\left(Z_{v_{B}} \in \Gamma_{A, B}\right)} .
$$


Proof. Start with (3) which, together with the definition of $\partial A$, implies that

$$
\pi(B)=\mathrm{E}_{\pi}\left(1_{\left(Z_{0} \in \partial A\right)} \mathrm{E}_{Z_{0}}\left(\sigma_{B}^{A}\right)\right) \leqslant \pi(\partial A) \sup _{y \in \partial A} \mathrm{E}_{y}\left(\sigma_{B}^{A}\right) .
$$

Let $z$ be any fixed element from $\partial A$. Then, by the strong Markov property of the Markov chain $Z$,

$$
\begin{aligned}
\mathrm{E}_{z}\left(\sigma_{B}^{A}\right) & =\mathrm{E}_{z}\left(\sigma_{B}^{A} 1_{\left(\tau_{B}<\tau_{A}\right)}\right)=\mathrm{E}_{z}\left(1_{\left(\tau_{B}<\tau_{A}\right)} \mathrm{E}_{z}\left(\sigma_{B}^{A} \mid \mathscr{F}_{\tau_{B}}\right)\right) \\
& =\mathrm{E}_{z}\left(1_{\left(\tau_{B}<\tau_{A}\right)} \mathrm{E}_{Z_{\tau_{B}}}\left(\sigma_{B}^{A}\right)\right) \leqslant P_{z}\left(\tau_{B}<\tau_{A}\right) \sup _{z \in B} \mathrm{E}_{z}\left(\sigma_{B}^{A}\right) .
\end{aligned}
$$

Now rewrite the total occupation time $\sigma_{B}^{A}$ of $B$ between two successive visits to $A$ as the sum over $k$ of the times spent by the 'stopped' process $\left\{Z_{n \wedge \tau_{A}}, n \geqslant 0\right\}$ in $B$ between the $k$ th and $(k+1)$ th consecutive visits of the set $\Gamma_{A, B}$. More precisely, define for $k \geqslant 1$,

$$
\begin{aligned}
\xi_{B}^{(0)} & =0, \quad \eta_{B}^{(1)}=\inf \left\{n \geqslant 0 ; Z_{n} \in \Gamma_{A, B}^{\prime \prime}\right\} ; \\
\xi_{B}^{(k)} & =\inf \left\{n \geqslant \eta_{B}^{(k)} ; \forall l \in\left[\eta_{B}^{(k)}, n\right), Z_{l} \in \Gamma_{A, B}^{\prime \prime} \text { and } Z_{n} \in \Gamma_{A, B}\right\} ; \\
\eta_{B}^{(k+1)} & =\inf \left\{n \geqslant \xi_{B}^{(k)} ; Z_{n} \in \Gamma_{A, B}^{\prime \prime}\right\} ; \\
\mu_{B}^{A} & =\max \left\{k \geqslant 0 ; \xi_{B}^{(k)}<\tau_{A}\right\}=\max \left\{k \geqslant 0 ; \eta_{B}^{(k)}<\tau_{A}\right\},
\end{aligned}
$$

with the usual convention $\inf \varnothing=+\infty$. Therefore, by the definition of $\mu_{B}^{A}$ and the strong Markov property at $\eta_{B}^{(k)}$, for any $y \in B$,

$$
\begin{aligned}
\mathrm{E}_{y}\left(\sigma_{B}^{A}\right) & =\mathrm{E}_{y}\left(\sum_{k=1}^{\mu_{B}^{A}} \sum_{n=\eta_{B}^{(k)}}^{\xi_{B}^{(k)}} 1_{\left(Z_{n} \in B\right)}\right)=\mathrm{E}_{y}\left(\sum_{k=1}^{\infty} 1_{\left(\mu_{B}^{A} \geqslant k\right)} \sum_{n=\eta_{B}^{(k)}}^{\xi_{B}^{(k)}} 1_{\left(Z_{n} \in B\right)}\right) \\
& =\sum_{k=1}^{\infty} \mathrm{E}_{y}\left(1_{\left(\mu_{B}^{A} \geqslant k\right)} \mathrm{E}\left(\sum_{n=\eta_{B}^{(k)}}^{\xi_{B}^{(k)}} 1_{\left(Z_{n} \in B\right)} \mid \mathscr{F}_{\eta_{B}^{k}}\right)\right) \\
& =\sum_{k=1}^{\infty} \mathrm{E}_{y}\left(1_{\left(\mu_{B}^{A} \geqslant k\right)} \mathrm{E}_{Z_{\eta_{B}^{(k)}}}\left(\sum_{n=0}^{\xi_{B}^{(1)}} 1_{\left(Z_{n} \in B\right)}\right)\right) \\
& \leqslant \sum_{k=1}^{\infty} P_{y}\left(\mu_{B}^{A} \geqslant k\right) \sup _{y^{\prime} \in B} \mathrm{E}_{y^{\prime}}\left(\sum_{n=0}^{\xi_{B}^{(1)}} 1_{\left(Z_{n} \in B\right)}\right) .
\end{aligned}
$$

Let us now have a closer look at the random time $\sum_{n=0}^{\xi_{B}^{(1)}} 1_{\left(Z_{n} \in B\right)}$ on the set $Z_{0} \in B$. Define new stopping times $\tau_{B}^{(k)} v_{B}^{(k)}$ by: 


$$
\begin{array}{ll}
\bar{\tau}_{B}^{(0)}=0, & \\
v_{B}^{(1)} \equiv v_{B}, & \tau_{B}^{(1)} \equiv \tau_{B}=\inf \left\{n \geqslant v_{B}^{(1)} ; Z_{n} \in B\right\}, \\
v_{B}^{(k+1)}=\inf \left\{n \geqslant \tau_{B}^{(k)} ; Z_{n} \notin B\right\}, & \tau_{B}^{(k+1)}=\inf \left\{n \geqslant v_{B}^{(k+1)} ; Z_{n} \in B\right\}, \\
\rho_{B}^{A}=\max \left\{k \geqslant 0 ; \tau_{B}^{(k)}<\tau_{A}\right\}, &
\end{array}
$$

(notice that $\rho_{B}^{A} \leqslant \sigma_{B}^{A}$ ). By this construction and the strong Markov property at $\tau_{B}^{(k)}$, for any $y^{\prime} \in B$,

$$
\begin{aligned}
\mathrm{E}_{y^{\prime}}\left(\sum_{n=0}^{\xi_{B}^{(1)}} 1_{\left(Z_{n} \in B\right)}\right) & =\sum_{k=0}^{\infty} \mathrm{E}_{y^{\prime}}\left(1_{\left(\rho_{B}^{\Gamma_{A}, B} \geqslant k\right)}\left(v_{B}^{(k+1)}-\tau_{B}^{(k)}\right)\right) \\
& \leqslant\left(1+\sum_{k=1}^{\infty} P_{y^{\prime}}\left(\rho_{B}^{\Gamma_{A, B}} \geqslant k\right)\right) \sup _{y^{\prime \prime} \in B} \mathrm{E}_{y^{\prime \prime}}\left(v_{B}\right) .
\end{aligned}
$$

Set $\bar{c}(B)=\sup _{y^{\prime} \in B} P_{y^{\prime}}\left(Z_{k} \in \Gamma_{A, B}^{\prime}, \forall k \in\left[v_{B}, \tau_{B}\right)\right)$. The strong Markov property at $\tau_{B}^{(k-1)}$ and the definition of $\rho_{B}^{\Gamma_{A, B}}, \tau_{B}^{(k)}$ imply that for any $y^{\prime} \in B$ and any $k \geqslant 1$,

$$
\begin{aligned}
P_{y^{\prime}}\left(\rho_{B}^{\Gamma_{A, B}} \geqslant k\right) & =P_{y^{\prime}}\left(\tau_{B}^{k}<\tau_{\Gamma_{A, B}}\right)=P_{y^{\prime}}\left(Z_{n} \in\left(\Gamma_{A, B}\right)^{c}, \forall n \in\left[v_{B}^{(l)}, \tau_{B}^{(l)}\right), \forall l \in[1, k]\right) \\
& \leqslant \bar{c}(B) P_{y^{\prime}}\left(Z_{n} \in\left(\Gamma_{A, B}\right)^{c}, \forall n \in\left[v_{B}^{(l)}, \tau_{B}^{(l)}\right), \forall l \in[1, k-1]\right) \leqslant(\bar{c}(B))^{k} .
\end{aligned}
$$

Also

$$
\begin{aligned}
1-\bar{c}(B) & =\inf _{y^{\prime} \in B} P_{y^{\prime}}\left(\exists k_{0} \in\left[v_{B}, \tau_{B}\right) ; Z_{k_{0}} \notin \Gamma_{A, B}^{\prime}\right) \\
& \geqslant \inf _{y^{\prime} \in B} P_{y^{\prime}}\left(Z_{v_{B}} \notin \Gamma_{A, B}^{\prime}\right)=\inf _{y^{\prime} \in B} P_{y^{\prime}}\left(Z_{v_{B}} \in \Gamma_{A, B}\right) .
\end{aligned}
$$

Hence,

$$
1+\sum_{k=1}^{\infty} \sup _{y^{\prime} \in B} P_{y^{\prime}}\left(\rho_{B}^{\Gamma_{A, B}} \geqslant k\right) \leqslant 1+\frac{\bar{c}(B)}{1-\bar{c}(B)} \leqslant \frac{1}{\inf _{y^{\prime} \in B} P_{y^{\prime}}\left(Z_{v_{B}} \in \Gamma_{A, B}\right)} .
$$

Substituting this in (13)

$$
\sup _{y^{\prime} \in B} \mathrm{E}_{y^{\prime}}\left(\sum_{n=0}^{\xi_{B}^{(1)}} 1_{\left(Z_{n} \in B\right)}\right) \leqslant \frac{\sup _{y^{\prime \prime} \in B} \mathrm{E}_{y^{\prime \prime}}\left(v_{B}\right)}{\inf _{y^{\prime} \in B} P_{y^{\prime}}\left(Z_{v_{B}} \in \Gamma_{A, B}\right)} .
$$

The remaining term $\sum_{k=1}^{\infty} \mathrm{E}_{y}\left(1_{\left(\mu_{B}^{A} \geqslant k\right)}\right)$ in the right-hand side of (11) can be estimated using the same idea that led to (14). For any $k \geqslant 1$ and any $y \in B$,

$$
P_{y}\left(\mu_{B}^{A} \geqslant k\right)=P_{y}\left(Z_{n} \in A^{c}, \forall n \in\left[\xi_{B}^{(l)}, \eta_{B}^{(l+1)}\right), \forall l \in[1, k-1]\right) \leqslant(\overline{\bar{c}}(B))^{k-1},
$$

where $\overline{\bar{c}}(B)=\sup _{y \in B} P_{y}\left(Z_{n} \in A^{c}, \forall n \in\left[\xi_{B}^{(1)}, \eta_{B}^{(2)}\right)\right)$. Therefore, applying again the strong Markov property at $\xi_{B}^{(1)}$,

$$
\sum_{k=1}^{\infty} P_{y}\left(\mu_{B}^{A} \geqslant k\right) \leqslant \frac{1}{1-\overline{\bar{c}}(B)} \leqslant \frac{1}{\inf _{y \in \Gamma_{A, B}} P_{y}\left(\tau_{A}<\tau_{\Gamma_{A, B}^{\prime \prime}}\right)} .
$$

Combining (8), (9), (11), (15) and (17) we obtain (7). 
We now obtain some useful bounds for the quantities appearing in (7). An example of their applications will be given later in this paper.

Remark 6. Suppose there exist an integer $m$ and $\gamma>0$ such that $\inf _{z \in B} P_{z}\left(v_{B} \leqslant m\right) \geqslant \gamma$. Then

$$
\sup _{z \in B} \mathrm{E}_{z}\left(v_{B}\right) \leqslant \frac{m}{\gamma}
$$

Proof. The bound is an immediate consequence of the Markov property and of the following:

Fact. Let $\zeta$ be a random variable taking values in $\{1,2, \ldots\}$. Suppose there exist an integer $m \geqslant 1$ and $\gamma>0$ such that for any $n \geqslant 0$,

$$
P(\zeta \leqslant n+m \mid \zeta>n) \geqslant \gamma .
$$

Then $\mathrm{E}(\zeta) \leqslant m / \gamma$.

Finally, the probabilities appearing in (7) can sometimes be estimated using the following result.

Lemma 1. Let $A$ be a finite subset of $S$ and let $D$ be any subset of $A^{c}$. Suppose that, for some $z \in(A \cup D)^{c}$ and a non-negative function $g$, the process $\left\{g\left(Z_{n \wedge \tau_{A}}\right), n \geqslant 0\right\}$ is a $\mathrm{P}_{\mathrm{z}^{-}}$ supermartingale. Suppose also that there exists a positive constant $\underline{d}=\underline{d}(D)$ such that $P_{z^{-}}$ a.s. $g\left(Z_{\tau_{D}}\right) \geqslant \underline{d}$. Then the following two statements hold:

(i)

$$
P_{z}\left(\tau_{A}>\tau_{D}\right) \leqslant \frac{g(z)}{\underline{d}}
$$

(ii)

$$
P_{z}\left(\tau_{A}<\tau_{D}\right) \geqslant \frac{d}{\underline{d}}-g(z)
$$

Proof. The recurrence of the Markov chain $\left\{Z_{n}, n \geqslant 0\right\}$ implies that the random time $\tau_{A} \wedge \tau_{D}$ is finite. Then, by Fatou's lemma applied to the positive sequence $\left\{g\left(Z_{n \wedge \tau_{A}} \wedge \tau_{D}\right)\right.$, $n \geqslant 0\}, g(z) \geqslant \mathrm{E}_{z}\left(g\left(Z_{\tau_{A} \wedge \tau_{D}}\right)\right)$. The proof is concluded by observing that

$$
\mathrm{E}_{z}\left(g\left(Z_{\tau_{A} \wedge \tau_{D}}\right)\right) \geqslant \mathrm{E}_{z}\left(g\left(Z_{\tau_{D}}\right) 1_{\left(\tau_{A}>\tau_{D}\right)}\right)
$$

and using the assumptions on $g$.

\subsection{Lower bounds for stationary distributions}

Theorem 3. Let $A$ be a finite subset of $S$ and $B$ be a subset of $A^{c}$ such that $\Gamma_{A, B}$ is non-empty and for any $z \in B, P_{z}\left(\tau_{A}=1\right)=0$. Then for any $z^{\prime} \in \partial A$, the following lower bound holds: 


$$
\pi(B) \geqslant \frac{\pi\left(z^{\prime}\right) P_{z^{\prime}}\left(\tau_{\Gamma_{A, B}^{\prime \prime}}<\tau_{A}\right) \inf _{z \in B} \mathrm{E}_{z}\left(v_{B}\right)}{\sup _{z \in \Gamma_{A, B}} P_{z}\left(\tau_{A}<\tau_{\Gamma_{A, B}^{\prime \prime}}\right) \sup _{z \in B} P_{z}\left(Z\left(v_{B}\right) \in \Gamma_{A, B}\right)}
$$

(by definition, $\mathrm{E}_{z}\left(v_{B}\right) \geqslant 1$ for any $z \in B$ ).

Proof. The proof is very similar to that of Theorem 2. Let $z^{\prime}$ be any state in $\partial A$. The application of the strong Markov property at $\tau_{B}, \tau_{\left\{z^{\prime}\right\}}$ gives

$$
\pi(B) \geqslant \pi\left(z^{\prime}\right) \mathrm{E}_{z^{\prime}}\left(\sigma_{B}^{A}\right)=\pi\left(z^{\prime}\right) \mathrm{E}_{z^{\prime}}\left(\sigma_{B}^{A} 1_{\left(\tau_{B}<\tau_{A}\right)}\right) \geqslant \pi\left(z^{\prime}\right) P_{z^{\prime}}\left(\tau_{\Gamma_{A, B}^{\prime \prime}}<\tau_{A}\right) \inf _{z \in B} \mathrm{E}_{z}\left(\sigma_{B}^{A}\right) .
$$

Let the random times $\xi_{B}^{(k)}, \eta_{B}^{(k)}, v_{B}^{(k)}, \tau_{B}^{(k)}, \mu_{B}^{A}$ and $\rho_{B}^{A}$ be defined as in (10), (12). From the definitions of $\Gamma_{A, B}^{\prime \prime}, \Gamma_{A, B}$ and the strong Markov property of the Markov chain $Z$ as in (11), (13), for any $z \in B$,

$$
\begin{aligned}
\mathrm{E}_{z}\left(\sigma_{B}^{A}\right) & \geqslant \sum_{k=1}^{\infty} P_{z}\left(\mu_{B}^{A} \geqslant k\right) \inf _{z \in B} \mathrm{E}_{z}\left(\sum_{n=0}^{\xi_{B}^{(1)}} 1_{\left(Z_{n} \in B\right)}\right) \\
& \geqslant \sum_{k=1}^{\infty} P_{z}\left(\mu_{B}^{A} \geqslant k\right)\left(1+\inf _{z \in B} \mathrm{E}_{z}\left(\rho_{B}^{\Gamma_{A, B}}\right)\right) \inf _{z \in B} \mathrm{E}_{z}\left(v_{B}\right) .
\end{aligned}
$$

Set $\underline{c}(B)=\inf _{z^{\prime} \in B} P_{z^{\prime}}\left(Z\left[v_{B}, \tau_{B}\right) \in \Gamma_{A, B}^{\prime}\right)$. Notice that $\underline{c}(B)<1$. Again, as in the proof of lower bounds, the strong Markov property at $\tau_{B}^{(k-1)}$ and the definition of $\rho_{B}^{\Gamma_{A, B}}, \tau_{B}^{(k)}$ imply that

$$
1+\inf _{z \in B} \mathrm{E}_{z}\left(\rho_{B}^{\Gamma_{A, B}}\right) \geqslant 1+\frac{\underline{c}(B)}{1-\underline{c}(B)} \geqslant \frac{1}{\sup _{z \in B} P_{z}\left(Z_{v_{B}} \in \Gamma_{A, B}\right)} .
$$

Let us now find a bound for $\sum_{k=1}^{\infty} P_{z}\left(\mu_{B}^{A} \geqslant k\right)$ from (23). Similarly to (16), for any $k \geqslant 1$ and any $z \in B$,

$$
P_{z}\left(\mu_{B}^{A} \geqslant k\right)=P_{z}\left(Z\left[\xi_{B}^{l}, \eta_{B}^{l+1}\right) \in A^{c}, \forall l \leqslant k-1\right) \geqslant(\underline{\underline{c}}(B))^{k-1},
$$

where $\underline{\underline{c}}(B)=\inf _{z \in B} P_{z}\left(Z\left[\xi_{B}^{(1)}, \eta_{B}^{(2)}\right) \in A^{c}\right)$. Hence

$$
\sum_{k=1}^{\infty} P_{z}\left(\mu_{B}^{A} \geqslant k\right) \geqslant \frac{1}{1-\underline{\underline{c}}(B)} \geqslant \frac{1}{\sup _{z \in \Gamma_{A, B}} P_{z}\left(\tau_{A}<\tau_{\Gamma_{A, B}^{\prime \prime}}\right)},
$$

which, together with (22)-(24), proves (21).

The following counterpart of Lemma 1 indicates a way to estimate the probabilities appearing in (21).

Lemma 2. Let $A$ be a finite subset of $S$ and let $D$ be some subset of $A^{c}$. Suppose, for some $z \in(A \cup D)^{c}$ and a non-negative function $\mathrm{g}$, that:

(a) The process $\left\{g\left(Z_{n \wedge \tau_{A} \wedge \tau_{D}}\right), n \geqslant 0\right\}$ is a $P_{z}$-submartingale.

(b) On $\left\{\tau_{A} \wedge \tau_{D}>n\right\}$ the family $\left\{g\left(Z_{n \wedge \tau_{A} \wedge \tau_{D}}\right), n \geqslant 0\right\}$ is $P_{z}$-a.s. bounded.

Then the following two statements hold: 
(i) If there exist positive constants $\bar{d}=\bar{d}(D), a=a(A)$ such that $g(z)>a$ and, $P_{z}$-a.s.,

$$
g\left(Z_{\tau_{D}}\right) \leqslant \bar{d}, \quad g\left(Z_{\tau_{A}}\right) \leqslant a,
$$

then

$$
P_{z}\left(\tau_{A}>\tau_{D}\right) \geqslant \frac{g(z)-a}{\bar{d}} .
$$

(ii) If there exist positive constants $\underline{d}=\underline{d}(D), a=a(A)$ such that

$$
g\left(Z_{\tau_{A}}\right) \leqslant a<\underline{d} \leqslant g\left(Z_{\tau_{D}}\right) \quad P_{z} \text {-a.s. }
$$

then

$$
P_{z}\left(\tau_{A}<\tau_{D}\right) \leqslant \frac{\mathrm{E}_{z}\left(g\left(Z_{\tau_{D}}\right)\right)-g(z)}{\underline{d}-a} .
$$

Proof. The proof proceeds similarly to that of Lemma 1. The submartingality assumption implies that, for any $n \geqslant 0$,

$$
g(z)=g\left(Z_{0}\right) \leqslant \mathrm{E}_{z}\left(g\left(Z_{n \wedge \tau_{A} \wedge \tau_{D}}\right)\right)=\mathrm{E}_{z}\left(g\left(Z_{n}\right) 1_{\left(n<\tau_{A} \wedge \tau_{D}\right)}\right)+\mathrm{E}_{z}\left(Z_{\tau_{A} \wedge \tau_{D}} 1_{\left(n \geqslant \tau_{A} \wedge \tau_{D}\right)}\right) .
$$

Boundedness of $g\left(Z_{n \wedge \tau_{A} \wedge \tau_{D}}\right)$, the recurrence of $\left\{Z_{n}, n \geqslant 0\right\}$, the dominated convergence theorem applied to $\mathrm{E}_{z}\left(g\left(Z_{n}\right) 1_{\left(n<\tau_{A} \wedge \tau_{D}\right)}\right)$, and the monotone convergence theorem applied to another term show that

$$
g(z) \leqslant \mathrm{E}_{z}\left(g\left(Z_{\tau_{A} \wedge \tau_{D}}\right)\right)
$$

But trivially,

$$
\begin{aligned}
g(z) \leqslant \mathrm{E}_{z}\left(g\left(Z_{\tau_{A} \wedge \tau_{D}}\right)\right) & =\mathrm{E}_{z}\left(g\left(Z_{\tau_{D}}\right)\right)-\mathrm{E}_{z}\left(\left(g\left(Z_{\tau_{D}}\right)-g\left(Z_{\tau_{A}}\right)\right) 1_{\left(\tau_{A}<\tau_{D}\right)}\right) \\
& \leqslant \mathrm{E}_{z}\left(g\left(Z_{\tau_{A}}\right)\right)+\mathrm{E}_{z}\left(g\left(Z_{\tau_{D}}\right) 1_{\left(\tau_{A}>\tau_{D}\right)}\right) .
\end{aligned}
$$

Inequalities (26) and (27) now follow immediately from the second and the first lines in (28) respectively and the assumptions of the lemma.

\section{Stationary measures for non-negative processes with asymptotically small drifts}

In this section we investigate the invariant measures of some non-negative Markov chains with asymptotically small drifts. Let $(\Omega, \mathscr{F}, P)$ be a probability space with a filtration $\left\{\mathscr{F}_{n}\right\}_{n \geqslant 0}$. Let $S$ be a countable unbounded set of $\mathbb{R}_{+}$such that its intersection with any compact subset of $\mathbb{R}_{+}$is finite. We are given a discrete-time $\left\{\mathscr{F}_{n}\right\}$-adapted non-negative irreducible aperiodic time-homogeneous Markov chain $\left\{X_{n}, n \geqslant 0\right\}$ taking values in $S$. We assume that it has bounded jumps, that is, there exists a positive constant $K$ such that for all $n \geqslant 0,\left|X_{n+1}-X_{n}\right| \leqslant K$. As usual, for any subset $F$ of $S, \tau_{F}=\inf \left\{n>0 ; X_{n} \in F\right\}$. For all $a \geqslant 0$ the symbol $\tau_{a}$ will stand for $\tau_{a}=\tau_{[0, a]}$. 
Theorem 4. Suppose the Markov chain $X$ has asymptotically small drift in the following sense: there exist constants $A>0, \varepsilon>0, a \geqslant 0$ such that for any $n \geqslant 0$, whenever $x>A$,

$$
\mathrm{E}\left(X_{n+1}-X_{n} \mid \mathscr{F}_{n}\right) \leqslant-\varepsilon \delta\left(X_{n}\right)\left(X_{n}\right)^{-a}, P_{x} \text {-a.s. } \quad \text { on }\left\{\tau_{A}>n\right\},
$$

where $\delta(x)=\mathrm{E}_{x}\left(\left(X_{1}-x\right)^{2}\right)$. Suppose also there is a positive constant $\mu$ such that

$$
\liminf _{x \rightarrow \infty} P_{x}\left(X_{1}-x<-\mu\right)>0 .
$$

Then the Markov chain is recurrent and the following statements hold:

(i) If $a<1$, then for any positive $\varepsilon_{0}<2 \varepsilon /(1-a)$,

$$
\int_{S} \mathrm{e}^{\varepsilon_{0} x^{1-a}} \pi(\mathrm{d} x)<\infty,
$$

and there exists a positive constant $c$ such that for all sufficiently large $b$,

$$
\pi([b, b+K)) \leqslant c \mathrm{e}^{-\varepsilon_{0} b^{1-a}} .
$$

(ii) If $a=1$, then for any positive $\varepsilon_{0}<2 \varepsilon$,

$$
\int_{S} x^{\varepsilon_{0}-1} \pi(\mathrm{d} x)<\infty,
$$

and there exists a positive constant $c$ such that for all sufficiently large $b$,

$$
\pi([b, b+K)) \leqslant c b^{-\varepsilon_{0}} .
$$

(iii) If $a>1$, then for any positive $\varepsilon_{0}$.

$$
\int_{S} \frac{\log ^{-\varepsilon_{0}-1} x}{x} \pi(\mathrm{d} x)<\infty,
$$

and there exists a positive constant $c$ such that for all sufficiently large $b$,

$$
\pi([b, b+K)) \leqslant c \log ^{\varepsilon_{0}} b .
$$

Remark 7. Since for any $\mu>0, \delta(x) \geqslant \mu^{2} P_{x}\left(X_{1}-x<-\mu\right)$, then (30) implies that $\liminf _{x \rightarrow \infty} \delta(x)>0$.

Remark 8. As will be seen below, the proof of integrability results only needs the condition $\lim \inf _{x \rightarrow \infty} \delta(x)>0$ instead of (30).

Proof. Let $\varepsilon_{0}$ be any fixed number satisfying the corresponding conditions of the theorem. For any $\varepsilon>0$, let the functions $g_{\varepsilon}, f_{\varepsilon}$ be defined by

$$
g_{\varepsilon}(x)= \begin{cases}\int_{0}^{x} \mathrm{e}^{\varepsilon s^{1-a}} \mathrm{~d} s, & \text { if } a<1, \\ x^{\varepsilon+1}, & \text { if } a=1, \\ x \log ^{-\varepsilon} x, & \text { if } a>1 .\end{cases}
$$




$$
f_{\varepsilon}(x)= \begin{cases}x^{-a} \mathrm{e}^{\varepsilon x^{1-a}} \delta(x), & \text { if } a<1, \\ x^{\varepsilon-1}, & \text { if } a=1, \\ x^{-1} \log ^{-\varepsilon-1} x, & \text { if } a>1\end{cases}
$$

It is easy then to see that there exists a positive $a_{0}$ such that for all $a>a_{0}$, whenever $x>a$, the process $\left\{g_{\varepsilon_{0}}\left(X_{n \wedge \tau_{a}}\right), n \geqslant 0\right\}$ is a $P_{x}$-supermartingale. Let us show this, for instance, in the case $a<1$. By Taylor's formula of second order, there exists a random variable $\xi_{n}$ such that

$$
\begin{aligned}
& \mathrm{E}\left(g_{\varepsilon_{0}}\left(X_{n+1}\right)-g_{\varepsilon_{0}}\left(X_{n}\right) \mid \mathscr{F}_{n}\right) \leqslant \mathrm{e}^{\varepsilon_{0} x^{1-a}} \mathrm{E}\left(X_{n+1}-X_{n} \mid \mathscr{F}_{n}\right) \\
& +\frac{\varepsilon_{0}(1-a)}{2} \mathrm{e}^{\varepsilon_{0} x^{1-a}} X_{n}^{-a} \delta\left(X_{n}\right)+\frac{1}{6} \mathrm{E}\left(g_{\varepsilon_{0}}^{\prime \prime}\left(\xi_{n}\right)\left|X_{n+1}-X_{n}\right|^{3} \mid \mathscr{F}_{n}\right) \\
& \quad \leqslant-f_{\varepsilon_{0}}\left(X_{n}\right) \liminf _{x \rightarrow \infty} \delta(x)+o\left(f_{\varepsilon_{0}}\left(X_{n}\right)\right) .
\end{aligned}
$$

Hence there exists a positive constant $c_{1}$ such that for all $a>a_{0}$, whenever $x>a$,

$$
\mathrm{E}\left(g_{\varepsilon_{0}}\left(X_{n+1}\right)-g_{\varepsilon_{0}}\left(X_{n}\right) \mid \mathscr{F}_{n}\right) \leqslant-c_{1} f_{\varepsilon_{0}}\left(X_{n}\right) P_{x} \text {-a.s. } \quad \text { on }\left\{\tau_{a}>n\right\} .
$$

This, Remark 7 and Theorem 1 prove the integrability results of the theorem. By Proposition 5.3 of Asmussen (1987) it also follows that the Markov chain $\left\{X_{n}, n \geqslant 0\right\}$ is recurrent.

Let us now prove the upper bounds. For all sufficiently large $b>a_{0} \vee 2 K$, set $A=\left(0, a_{0}\right), B=[b, b+K)$. Then, $\Gamma_{A, B}=[b-K, b), \quad \Gamma_{A, B}^{\prime}=[b+K, b+2 K), \quad \Gamma_{A, B}^{\prime \prime}=$ $[b, \infty)$ and, immediately, for all $x \in\left(a_{0}, b\right)$,

$$
g_{\varepsilon_{0}}(b) \leqslant g_{\varepsilon_{0}}\left(X_{\mathrm{T}_{\Gamma \ddot{A}, B}}\right)<g(b+K), \quad \text { with } P_{x} \text {-probability } 1 .
$$

Notice that (30) implies that there exist positive constants $n_{0}, p_{0}$ such that for any $x \in \Gamma_{A, B}$ there exists $x^{\prime} \in[b-2 K, b-K)$ satisfying ${ }_{(A \cup B)} P_{x, x^{\prime}}^{n_{0}}>p_{0}$. Therefore, by Lemma 1 there exist positive constants $c_{1}, c_{2}$ such that for all sufficiently large $b$,

$$
\begin{aligned}
\inf _{x \in \Gamma_{A, B}} P_{x}\left(\tau_{A}<\tau_{\Gamma_{A, B}^{\prime \prime}}\right) & \geqslant p_{0} \frac{g_{\varepsilon_{0}}(b)-\sup _{x \in[b-2 K, b-K)} g_{\varepsilon_{0}}(x)}{g(b+K)} \leqslant p_{0} \frac{g_{\varepsilon_{0}}(b)-g_{\varepsilon_{0}}(b-K)}{g_{\varepsilon_{0}}(b+K)} \\
& \leqslant p_{0} \frac{\inf _{[b-K, b]} g_{\varepsilon_{0}}^{\prime}}{g_{\varepsilon_{0}}(b+K)}, \\
\sup _{x \in \partial A} P_{x}\left(\tau_{\Gamma_{A, B}^{\prime \prime}}<\tau_{A}\right) & \leqslant \sup _{y \in}\left(a_{0}-K, a_{0}\right) P_{y}\left(\tau_{A}>\tau_{\Gamma_{A, B}^{\prime \prime}}\right) \leqslant \frac{g_{\varepsilon_{0}}\left(a_{0}\right)}{g_{\varepsilon_{0}}(b)} .
\end{aligned}
$$

Let us denote the value of the limit in (30) by $p_{2}$. Then because of (30), there exists an $n_{1}>K / \mu$ such that for any $z \in B, P_{z}\left(Z\left(v_{B}\right) \in \Gamma_{A, B}\right) \geqslant P_{z}\left(Z\left(v_{B}\right) \in \Gamma_{A, B}, \quad v_{B} \leqslant n_{1}\right) \geqslant p_{1}$, where $p_{1}=p_{2}^{K / \mu}$. Furthermore, by Remark 6 it follows from these bounds that $\sup _{x \in B} \mathrm{E}_{x}\left(v_{B}\right) \leqslant n_{1} / p_{1}$. Substituting these bounds and (33) in Theorem 2 gives the desired upper bounds.

Remark 9. Theorem 4 implies that in the case $a<1$ and $a=1, \varepsilon>\frac{1}{2}$ the Markov chain $\left\{X_{n}, n \geqslant 0\right\}$ is positive recurrent. 
Theorem 5. If in the conditions of Theorem 4 we replace (30) with the assumption that the Markov chain $\left\{X_{n}, n \geqslant 0\right\}$ is recurrent and there exist constants $A>0, \varepsilon>0, a \geqslant 0$ such that for any $n \geqslant 0$, whenever $x>A$,

$$
\mathrm{E}\left(X_{n+1}-X_{n} \mid \mathscr{F}_{n}\right) \geqslant-\varepsilon \delta\left(X_{n}\right)\left(X_{n}\right)^{-a} P_{x} \text {-a.s. } \quad \text { on }\left\{\tau_{A}>n\right\},
$$

then:

(i) If $a<1$, then for any positive $\varepsilon_{0}>2 \varepsilon /(1-a)$,

$$
\int_{S} \mathrm{e}^{\varepsilon_{0} x^{1-a}} \pi(\mathrm{d} x)=\infty
$$

and there exists a positive constant $c$ such that for all sufficiently large $b$,

$$
\pi([b, b+K)) \geqslant c \mathrm{e}^{-\varepsilon_{0} b^{1-a}} .
$$

(ii) If $a=1$, then for any positive $\varepsilon_{0}>2 \varepsilon$,

$$
\int_{S} x^{\varepsilon_{0}-1} \pi(\mathrm{d} x)=\infty
$$

and there exists a positive constant $c$ such that for all sufficiently large $b$,

$$
\pi([b, b+K)) \geqslant c b^{-\varepsilon_{0}} .
$$

(iii) If $a>1$, then for any positive $\varepsilon_{0}$,

$$
\int_{S} \frac{\log ^{\varepsilon_{0}-1} x}{x} \pi(\mathrm{d} x)=\infty
$$

and there exists a positive constant $c$ such that for all sufficiently large $b$,

$$
\pi([b, b+K)) \geqslant c \log ^{-\varepsilon_{0}} b .
$$

Remark 10. Notice that in fact the conditions on the boundedness of increments of the Markov chain $\left\{X_{n}, n \geqslant 0\right\}$ can be relaxed. For instance, for the integrability (nonintegrability) results it suffices to assume that there exists a positive constant $\gamma>2$ such that

$$
\sup _{x \in S} \mathrm{E}_{x}\left(\left|X_{1}-x\right|^{\gamma}\right)<\infty .
$$

In this way one can recover Lemma 3 of Borovkov et al. (1992) from Statement (ii) of Theorem 4 (the case $a=1$ ). Moreover, statement (ii) of Theorem 5 completes it, giving the corresponding divergence result. As for estimates of the invariant measure, one can obtain similar results assuming simply the condition of lower boundedness of the increments by some constant $K^{\prime}$ and (35).

Remark 11. The previous remark and Theorems 4 and 5 suggest a way of estimating the stationary probabilities of some countable Markov chains in some situations. Suppose we are given an irreducible aperiodic recurrent Markov chain $\left\{Z_{n}, n \geqslant 0\right\}$ on some countable state space $S$ and a positive function $F$ (called a Lyapunov or test function) defined on $S$ such that 
the process $X=\left\{F\left(Z_{n}\right), n \geqslant 0\right\}$ satisfies the conditions of Theorem 4 or 5 and Remark 10 . Then the latter results permit one to estimate the stationary measures of the sets $F^{-1}(b, b+K)=\{z \in S ; F(z) \in(b, b+K)\}$ for all sufficiently large $b$ and give sufficient conditions of (non-)integrability of certain functions $f$ with respect to the stationary measure.

Proof of Theorem 5. Let $\varepsilon_{0}$ be any constant satisfying the conditions of the theorem and $g_{\varepsilon_{0}}$ be defined by (31). It can then be seen that there exists a positive $a_{0}$ such that for all $a>a_{0}$ whenever $x>a$, the process $\left\{g_{\varepsilon_{0}}\left(X_{n \wedge \tau_{a}}\right), n \geqslant 0\right\}$ is a $P_{x}$-submartingale. Moreover, there exists a positive constant $c_{1}$ such that for all $a>a_{0}$, whenever $x>a$,

$$
\mathrm{E}\left(g_{\varepsilon_{0}}\left(X_{n+1}\right)-g_{\varepsilon_{0}}\left(X_{n}\right) \mid \mathscr{F}_{n}\right) \leqslant c_{1} f_{\varepsilon_{0}}\left(X_{n}\right) P_{x} \text {-a.s. } \quad \text { on }\left\{\tau_{a}>n\right\},
$$

where the function $f_{\varepsilon_{0}}$ is defined in (32). Let us now consider the function $h=g_{\varepsilon_{1}}$ with some $\varepsilon_{1}<\varepsilon_{0}$ satisfying the conditions imposed on $\varepsilon$ of the theorem. Then we can see that there exists a positive constant $a_{1}$ such that for all $a>a_{1}$ whenever $x>a$, the process $\left\{h\left(X_{n \wedge \tau_{a}}\right)\right.$, $n \geqslant 0\}$ is a $P_{x}$-submartingale. This, (36), Theorem $1^{\prime}$ and Remark 4 imply the desired divergence. The proof of other statements of the theorem can be carried out by using Theorem 3 and Lemma 2 and is left to the reader.

Remark 12. Theorem 5 implies that in the case $a>1$ and $a=1, \varepsilon<1 / 2$ the Markov chain $\left\{X_{n}, n \geqslant 0\right\}$ is null recurrent.

Remark 13. The bounds in statements (i) (case $0<a<1$ ) of Theorems 4 and 5 were recently obtained by different methods in Menshikov and Popov (1995).

\section{Reflected random walks in a quadrant}

\subsection{Statement of the results}

Let $\tilde{G}$ be the quadrant given by $\tilde{G}=\left\{(x, y) \in \mathbb{R}^{2} ; x \geqslant 0, y \geqslant 0\right\}$. The two sides of the quadrant are denoted by $\partial \tilde{G}_{1}$ and $\partial \tilde{G}_{2}$, where $\partial \tilde{G}_{1}=\{(x, y) \in \tilde{G} ; x \neq 0, y=0\}$ and $\partial \tilde{G}_{2}=\{(x, y) \in \tilde{G} ; y \neq 0, x=0\}$. The interior of $\tilde{G}$ is referred to as $\tilde{G}^{0}$ and the boundary of the wedge (i.e. $\partial \tilde{G}_{1} \cup \partial \tilde{G}_{2} \cup(0,0)$ ) will be denoted by $\partial \tilde{G}$. We consider the discrete-time homogeneous irreducible aperiodic $\left\{\mathscr{F}_{n}\right\}$-adapted Markov chain $\left\{\tilde{Z}_{n}, n \geqslant 0\right\}$ taking values in $\mathbb{Z}_{+}^{2}$ defined inductively for $n \geqslant 0$ by

$$
\tilde{Z}_{n+1}=\tilde{Z}_{n}+Y_{n}^{(0)} 1_{\left(Z_{n} \in \tilde{G}^{0}\right)}+Y_{n}^{(1)} 1_{\left(\tilde{Z}_{n} \in \partial \tilde{G}_{1}\right)}+Y_{n}^{(2)} 1_{\left(\tilde{Z}_{n} \in \partial \tilde{G}_{2}\right)}+Y_{n}^{(3)} 1_{\left(\tilde{Z}_{n}=(0,0)\right)},
$$

where for each $l=0,1,2,3$ the random vectors $Y_{n}^{(l)}, n \geqslant 0$ are i.i.d. and have the following probability distributions:

$$
P\left(Y^{(l)}=(i, j)\right)=p_{i, j}^{(l)}, \quad i, j \geqslant-1, \quad p_{0,0}^{(l)}=0 .
$$

In order to keep the process in $\mathbb{Z}_{+}^{2}$ it is assumed that for all $i, j \geqslant-1, p_{i,-1}^{1}=$ $p_{-1, j}^{2}=p_{i,-1}^{3}=p_{-1, j}^{3}=0$. We also assume that $\gamma$ defined by 


$$
\gamma \equiv \sup \left\{\kappa ; \sum_{i, j}\left(|i|^{\kappa}+|j|^{\kappa}\right) p_{i, j}^{l}<\infty, \text { for all } 1=0,1,2\right\}>2,
$$

$\mathrm{E}\left(Y_{n}^{(0)}\right)=(0,0)$ and the covariance matrices (denoted $\left.A^{l}\right)$ of $Y_{n}^{(l)}$ are positive definite. The $n$ step transition probabilities ( $n$-step transition probabilities with taboo set $A$ ) of $\left\{Z_{n}, n \geqslant 0\right\}$ are denoted by $P_{z, z^{\prime}}^{n}, z, z^{\prime} \in \mathbb{Z}_{+}^{2}\left({ }_{A} P_{z, z^{\prime}}^{n}, z, z^{\prime} \in \mathbb{Z}_{+}^{2}\right)$.

Let us now introduce one useful transformation of the state space which permits us to simplify the presentation of the results. Namely, let $\Phi$ be any linear isomorphism of $\mathbb{R}^{2}$ such that the vector $\Phi\left(Y^{(0)}\right)$ has the unit covariance matrix. For instance, we can define $\Phi$ as in Aspandiiarov and Iasnogorodski (1997) (see formulae (13)-(14) therein). Set

$$
\begin{aligned}
G_{4} & =\Phi\left(\mathbb{Z}_{+}^{2}\right), \quad G=\Phi(\tilde{G}), \quad G^{0}=\Phi\left(\tilde{G}^{0}\right), \\
\partial G_{1} & =\Phi\left(\partial \tilde{G}_{1}\right), \quad \partial G_{2}=\Phi\left(\partial \tilde{G}_{2}\right), \quad \partial G=\Phi(\partial \tilde{G}) ;
\end{aligned}
$$

and denote the angle of the wedge $G$ by $\xi$. We also introduce the following family of nonnegative harmonic functions on $G \backslash(0,0)$. For any $\beta_{1}, \beta_{2} \in(-\pi / 2, \pi / 2)$ we set $\beta=$ $\left(\beta_{1}+\beta_{2}\right) / \xi$ and define the function $\Psi_{\beta_{1}, \beta_{2}}: G \rightarrow \mathbb{R}_{+}$in standard polar coordinates $(\rho, \theta)$ by

$$
\Psi_{\beta_{1}, \beta_{2}}(z)= \begin{cases}\psi_{\beta_{1}, \beta_{2}}^{1 / \beta}(z), & \text { if } \beta \neq 0, \\ \exp \left(\psi_{\beta_{1}, \beta_{2}}(z)\right), & \text { if } \beta=0,\end{cases}
$$

where the function $\psi_{\beta_{1}, \beta_{2}}$ is given by

$$
\psi_{\beta_{1}, \beta_{2}}(\rho, \theta)= \begin{cases}\rho^{\beta} \cos \left(\beta \theta-\beta_{1}\right), & \beta_{1}+\beta_{2} \neq 0, \rho>0, \theta \in[0, \xi], \\ \log \rho+\left|\tan \beta_{1}\right| \theta, & \beta_{1}+\beta_{2}=0, \rho>0, \theta \in[0, \xi], \\ 0, & \rho=0\end{cases}
$$

We can now define the crucial parameters for our study. Let $\alpha_{1}, \alpha_{2}$ be the angles between the vectors $\Phi\left(\mathrm{E}\left(Y_{n}^{1}\right)\right), \Phi\left(\mathrm{E}\left(Y_{n}^{2}\right)\right)$ and the inward normals to the corresponding axes $\partial G_{1}$ and $\partial G_{2}$, with positive angles towards the corner. By our assumptions on the transition mechanism of the Markov chain $\tilde{Z}$, the distributions of $Y^{1}, Y^{2}$ are non-degenerate so that the angles $\tilde{\alpha}_{1}, \tilde{\alpha}_{2}$ between the vectors $\mathrm{E}\left(Y_{n}^{(1)}\right), \mathrm{E}\left(Y_{n}^{(2)}\right)$ and the inward normals to the corresponding axes $\partial \tilde{G}_{1}$ and $\partial \tilde{G}_{2}$ are different from $-\pi / 2, \pi / 2$. Consequently, $\alpha_{1}$, $\alpha_{2} \in(-\pi / 2, \pi / 2)$. Define the function $\tilde{\Psi}$ on $\tilde{G}$ by

$$
\tilde{\Psi}(z)=\Psi_{\alpha_{1}, \alpha_{2}}(\Phi(z)), \quad z \in \tilde{G} .
$$

As is easy to see from the definitions of $\Phi$ and $\Psi_{\alpha_{1}, \alpha_{2}}$, there exists a positive constant $c \alpha_{1}, \alpha_{2}$ such that, for all $z_{1}, z_{2} \in \tilde{G}$,

$$
\left|\tilde{\Psi}\left(z_{1}\right)-\tilde{\Psi}\left(z_{2}\right)\right| \leqslant c_{\alpha_{1}, \alpha_{2}}\left|z_{1}-z_{2}\right| .
$$

Set

$$
\alpha=\frac{\alpha_{1}+\alpha_{2}}{\xi}
$$

and define a function $h$ on $\mathbb{R}_{+}$by 


$$
h(b)= \begin{cases}b^{1-\alpha}, & \text { if } \alpha \neq 0 \\ b / \log (b), & \text { if } \alpha=0 .\end{cases}
$$

The importance of the parameter $\alpha$ is explained by the following recurrence criterion for the Markov chain $\left\{\tilde{Z}_{n}, n \geqslant 0\right\}$ whose proof can be found in Aspandiiarov et al. (1996, Theorem 5) and Aspandiiarov and Iasnogorodski (1997, Remark 4).

Theorem 6. The Markov chain $\left\{\tilde{Z}_{n}, n \geqslant 0\right\}$ is recurrent if and only if $\alpha \geqslant 0$.

Throughout the rest of this paper we will suppose that $\alpha \geqslant 0$. Our main results describing the asymptotic behaviour of the stationary measure of the reflected random walk $\tilde{Z}$ in the case $\alpha \geqslant 0$ are stated as follows.

Theorem 7 (Integrability). For any $\mu>0$ and any integer $k \geqslant 1$,

$$
\begin{gathered}
\int_{\tilde{G}^{0}} \frac{|z|^{\alpha-2}}{\phi_{k}(\mu,|z|)} \tilde{\pi}(\mathrm{d} z)<\infty, \quad \int_{\tilde{G}^{0}} \frac{|z|^{\alpha-2}}{\phi_{k}(-\mu,|z|)} \tilde{\pi}(\mathrm{d} z)=\infty, \\
\int_{\left(\partial \tilde{G}_{1} \cup \partial \tilde{G}_{2}\right)} \frac{|z|^{\alpha-1}}{\phi_{k}(\mu,|z|)} \tilde{\pi}(\mathrm{d} z)<\infty, \quad \int_{\left(\partial \tilde{G}_{1} \cup \partial \tilde{G}_{2}\right)}|z|^{\alpha-1} \log _{k}^{\mu}(|z|) \tilde{\pi}(\mathrm{d} z)=\infty,
\end{gathered}
$$

where the functions $\phi_{k}: \mathbb{R} \times \mathbb{R}_{+} \rightarrow \mathbb{R}_{+}$are defined, for all sufficiently large $x$, by

$$
\phi_{k}(s, x)= \begin{cases}\log (x) \ldots \log _{k-1}(x) \log _{k}^{1+s}(x), & \text { if } k>1 \\ \log 1+s(x), & \text { if } k=1\end{cases}
$$

and $\log _{k}$ are iterated logarithms.

To simplify the presentation of the remaining results of this section the following is also assumed (see Remark 17):

Boundedness of increments. The increments $Z_{n+1}-Z_{n}$ are almost surely bounded, that is, there is a positive constant $K^{\prime}$ such that, with probability $1,\left|Y^{(l)}\right| \leqslant K^{\prime}$ for any $l=0,1,2,3$. Set

$$
K_{\alpha}=c_{\alpha_{1}, \alpha_{2}} \sqrt{2 K^{\prime}}
$$

where $c_{\alpha_{1}, \alpha_{2}}$ satisfies (41).

Theorem 8 (Local bounds). There exist positive constants $K, b_{0}, c_{1}$, and $c_{2}$ such that, for all $b>b_{0}$,

$$
c_{1} h(b) \leqslant \tilde{\pi}(b<\tilde{\Psi}(z) \leqslant b+K) \leqslant c_{2} h(b) \log (b) .
$$

Theorem 9 (Global bounds on the boundary $\partial \tilde{G})$. Let $\phi_{k}(\mu, b)$ be defined as in (46). Then, for any $\mu>0$ and for any integer $k \geqslant 1$, there exist positive constants $b_{1}, c_{3}, c_{4}$ such that for all $b>b_{1}$ : 
(i) If $\alpha>1$, then $\tilde{\pi}(\partial \tilde{G})<\infty$ and

$$
\tilde{\pi}(|z|>b, z \in \partial \tilde{G}) \leqslant c_{4} b^{1-\alpha} \phi_{k}(\mu, b) .
$$

(ii) If $\alpha \leqslant 1$, then

$$
c_{3} h(b) \leqslant \tilde{\pi}(|z|<b, z \in \partial \tilde{G}) \leqslant c_{4} b^{1-\alpha} \phi_{k}(\mu, b) .
$$

Theorem 10 (Global bounds in the interior $\tilde{G}^{0}$ ). For any $\mu>0$ and for any integer $k \geqslant 1$, there exist positive constants $b_{2}, c_{5}, c_{6}$ such that the following statements hold for all $b>b_{2}$ :

(i) If $\alpha \neq 2$, then

$$
c_{5} b h(b) \leqslant \tilde{\pi}\left(|z|>b, z \in \tilde{G}^{0}\right) \leqslant c_{6} b h(b) \log (b) .
$$

(ii) If $\alpha=2$, then

$$
c_{5} \log (b) \leqslant \tilde{\pi}\left(|z|<b, z \in \tilde{G}^{0}\right) \leqslant c_{6} \log ^{2}(b) .
$$

Remark 14. In the case $\alpha \in(1,2]$ the invariant measure of the boundary is finite, which means that the time which an excursion of the Markov chain outside the origin spends on the boundary has a finite mean. In other words, the embedded Markov chain constructed by observing the original Markov chain when it hits the boundary is ergodic. This result can be seen as a discrete-time analogue of the fact that the reflected Brownian motion with the same oblique reflection in the wedge $G$ is not a semimartingale (see Williams 1985).

Remark 15. The results of Theorems 8 and 10 in the case $\alpha \in[0,2)$ give almost the same asymptotics as those for the corresponding Brownian motion with oblique reflection in a wedge that can be derived from Theorem 6.1 in Williams (1985), giving an exact expression for the invariant measure of a reflected Brownian motion a wedge. In this sense Theorems 8 and 10 can be viewed as random walk analogues of Theorem 6.1 in Williams (1985). It should be said that our results provide bounds for the 'tails' of the stationary measure on the boundary which have no counterparts for reflected diffusions.

Remark 16. In Menshikov and Popov (1995) the following result related to (49) and (51) has been proved by a different method. If $\alpha>2$, then for any $\mu>0$ there exist positive constants $b_{0}, c_{1}, c_{2}$ such that for all $b>b_{0}$,

$$
c_{1} b^{2-\alpha-\mu} \leqslant \tilde{\pi}(|z|>b) \leqslant c_{2} b^{2-\alpha+\mu} .
$$

As a by-product of Theorem 7 we recover the following criterion of positive recurrence of the Markov chain $\left\{\tilde{Z}_{n}, n \geqslant 0\right\}$ from Aspandiiarov et al. (1996) and Aspandiiarov and Iasnogorodski (1997).

Corollary 1. The Markov chain $\left\{\tilde{Z}_{n}, n \geqslant 0\right\}$ is positive recurrent if and only if $\alpha>2$.

Before proving the results let us say a few words on the boundedness of increments 
restriction (the existence of $K^{\prime}$ ) which was imposed on the transition mechanism of the Markov chain $\tilde{Z}$.

Remark 17. If one does not impose the condition of boundedness of increments (existence of $K^{\prime}$ ), then Theorem 9 remains valid whereas Theorems 8 and 10 need some changes. Theorem 8 is valid only in the case $\alpha \geqslant 1$ and we make the following change: for any $\eta>0$ there exist positive constants $b_{0}, c_{1}, c_{2}$ such that

$$
c_{1} h(b) b^{-1 /(\gamma-1)-\eta} \leqslant \tilde{\pi}(b<\tilde{\Psi}(z) \leqslant b+K) \leqslant c_{2} h(b) \log (\mathrm{b}) .
$$

As far as Theorem 10 is concerned, its upper bounds and the lower bound in the case $\alpha \in[0,2]$ remain unchanged. But the lower bound in the case $\alpha>2$ is stated differently: for any $\eta>0$ there exist positive constants $b_{2}, c_{5}$ such that, for all $b>b_{2}$,

$$
c_{5} b^{2-\alpha-1 /(\gamma-1)-\eta} \leqslant \tilde{\pi}\left(|z|>b, z \in \tilde{G}^{0}\right) .
$$

Similarly, if one imposes the existence of exponential moments of the random vectors $Y_{n}^{(l)}$, then one can replace $b^{-1 /(\gamma-1)-\eta}$ in the lower bounds of (53) and (54) by $\log ^{-1}(b)$.

The proof of these claims can be found in Aspandiiarov and Iasnogorodski (1995).

We will prove Theorems $7-10$ by demonstrating the analogues of these results for the Markov chain $\left\{Z_{n}, n \geqslant 0\right\}$ defined as the image of the Markov chain $\left\{\tilde{Z}_{n}, n \geqslant 0\right\}$ under the linear isomorphism $\Phi$, the measure $\pi$ defined by $\pi(\cdot)=\tilde{\pi}\left(\Phi^{-1}(\cdot)\right)$ instead of $\tilde{\pi}$, the function $\Psi$ instead of $\tilde{\Psi}, G^{0}, \partial G, \partial G_{1}, \partial G_{2}, G_{4}$ instead of $\tilde{G}^{0}, \partial \tilde{G}, \partial \tilde{G}_{1}, \partial \tilde{G}_{2}, \mathbb{Z}_{+}^{2}$ respectively. The proof of the latter assertions will be based on the results of Section 2 .

The plan of the rest of the paper is as follows. First we prove the local bounds of Theorem 8 and show a technique for constructing sub- or supermartingales which will be used throughout the proofs. Then we will move on to the (non-)integrability assertions of Theorem 7. Once we prove these results, the remaining global bounds of Theorems 9-10 will be obtained easily as their consequences. Finally, in Appendix A we will prove some of auxiliary martingale results and in Appendix B we deal with some geometrical properties of the Markov chain $Z$.

\section{Proof of the main results}

\subsection{Preliminary results and notation}

An important role in the proof will be played by so-called 'monotonicity' property of $\psi_{\beta_{1}, \beta_{2}}$ : there exists a positive constant $c_{1}$ such that for any $z \in G \backslash(0,0),|z|>2$,

$$
\left.\begin{array}{ll}
\log |z|, & \text { if } \beta=0 \\
|z|^{\beta} \cos \left(\left|\beta_{1}\right| \vee\left|\beta_{2}\right|\right), & \text { if } \beta>0
\end{array}\right\} \leqslant \psi_{\beta_{1}, \beta_{2}}(z) \leqslant \begin{cases}c_{1} \log |z|, & \text { if } \beta=0 \\
|z|^{\beta}, & \text { if } \beta>0 .\end{cases}
$$

We need more notation. For any $\beta_{1}, \beta_{2} \in(-\pi / 2, \pi / 2), a>0$, we will denote by $F_{\beta ; a}$ the following set: 


$$
F_{\beta ; a}=\left\{z \in G ; \Psi_{\beta_{1}, \beta_{2}}(z) \leqslant a\right\} .
$$

Again for simplicity the index $\beta$ in $F_{\beta ; a}$ will be omitted if $\beta_{1}=\alpha_{1}, \beta_{2}=\alpha_{2}$, and we will simply write $F_{a}$. Let us also introduce a positive constant $c(\beta)$ given by

$$
c(\beta)= \begin{cases}\cos ^{-1 / \beta}\left(\left|\beta_{1}\right| \vee\left|\beta_{2}\right|\right), & \text { if } \beta \neq 0 \\ \exp \left(-\xi\left|\tan \beta_{1}\right|\right), & \text { if } \beta=0\end{cases}
$$

As usual, for any subset $F$ of $G$ we let $\tau_{F}=\inf \left\{n>0 ; Z_{n} \in F\right\}$. In particular, for each $a>0$, we will simplify the notation, writing $\tau_{\beta ; a}$ instead of $\tau_{F_{\beta ; a}}$ and $\tilde{\tau}_{a}$ instead of $\tau_{F_{a}^{c}}$. Similarly, we will write $\tilde{\tau}_{\beta ; a}$ instead of $\tau_{F_{\beta ; a}^{c}}$ and $\tilde{\tau}_{a}$ instead of $\tau_{F_{a}^{c}}$.

Lemma 3. Let $a_{0}$ be some positive constant and $\beta_{1}, \beta_{2} \in(-\pi / 2, \pi / 2)$.

(i) There exist $b_{0}>0, p_{0}>0, n_{0}>0$ such that for all $b>b_{0}$ and for any $z \in F_{\beta ; b+K} \backslash F_{\beta ; b}$, satisfying $p_{z^{\prime \prime}, z}>0$ for some $z^{\prime \prime} \in F_{\beta ; b}$, there is some $z^{\prime} \in F_{\beta ; b+K}^{c}$ such that

$$
F_{\beta ; b} P_{z, z^{\prime}}^{\left(n_{0}\right)}>p_{0} .
$$

(ii) There exist $b_{0}>0, p_{0}>0, n_{0}>0$ such that for all $b>b_{0}$ and $z \in \Gamma_{F_{\beta ; a_{0}}, F_{\beta ;},}$, there is some $z^{\prime} \in F_{\beta ; b-K}$ such that

$$
\left(F_{\beta ; b}^{c} \cup F_{\beta ; a_{0}}\right) P_{z, z^{\prime}}^{\left(n_{0}\right)}>p_{0} .
$$

The proof will be given in Appendix B.

Convention. Throughout the proof the quantities $c_{1}, c_{2}, c_{3}, \ldots$ will denote positive constants that do not depend on $b$. As usual, all sets and initial states appearing below are assumed to be subsets of the appropriate state spaces of the Markov chain under consideration. A similar convention is applied to all functions $f, g$.

\subsection{Lower local bounds of Theorem 8}

We will only prove the lower bounds for $K=K_{\alpha}$, which immediately provides the same bounds for an arbitrary $K \geqslant K_{\alpha}$.

Proposition 2. Let $\alpha \geqslant 0$. There is a positive constant $c$ such that for all sufficiently large a, $b$ satisfying $b>a$ and for any $z \in F_{b} \backslash F_{b-K_{a}}, P_{z}\left(\tau_{A}<\tilde{\tau}_{b}\right) \leqslant c / b$.

Proof. Let $\kappa>1$ be a sufficiently large number which will be defined later. Let us define the function $g$ by $g(z)=T\left(\psi_{\alpha_{1}, \alpha_{2}}\right)(z)$, where $T(x)=x^{\kappa}$ if $\alpha \neq 0$ and $T(x)=\exp (\kappa x)$ if $\alpha=0$.

Lemma 4. For any $a, b$ and $z$ satisfying the hypothesis of Proposition 2, the process $\left\{g\left(Z_{n \wedge \tau_{a}}\right), n \geqslant 0\right\}$ is a $P_{z}$-submartingale.

Proof. By the second-order Taylor formula for any function $f \in C^{3}$, 


$$
\begin{aligned}
f \circ \psi_{\beta_{1}, \beta_{2}}\left(Z_{n+1}\right) & -f \circ \psi_{\beta_{1}, \beta_{2}}\left(Z_{n}\right)=f^{\prime} \circ \psi_{\beta_{1}, \beta_{2}}\left(Z_{n}\right)\left(\nabla \psi_{\beta_{1}, \beta_{2}}\left(Z_{n}\right), \Delta_{n}\right) \\
& +\frac{1}{2} f^{\prime \prime} \circ \psi_{\beta_{1}, \beta_{2}}\left(Z_{n}\right)\left(\nabla \psi_{\beta_{1}, \beta_{2}}\left(Z_{n}\right), \Delta_{n}\right)^{2} \\
& +\frac{1}{2} f^{\prime} \circ \psi_{\beta_{1}, \beta_{2}}\left(Z_{n}\right) D^{2} \psi_{\beta_{1}, \beta_{2}}\left(Z_{n}, \Delta_{n}, \Delta_{n}\right)+R_{n}\left(Z_{n}, \Delta_{n}, f, \beta, 2\right),
\end{aligned}
$$

where

$$
R_{n}\left(Z_{n}, \Delta_{n}, f, \beta, 2\right)=\frac{1}{2 !} \int_{0}^{1} \frac{\mathrm{d}^{3}}{\mathrm{~d} t^{3}}\left\{f \circ \psi_{\beta_{1}, \beta_{2}}\left(Z_{n}+t \Delta_{n}\right)\right\}(1-t)^{2} \mathrm{~d} t .
$$

In the proof of Lemma 11 (see Aspandiiarov and Iasnogorodski 1997) it was shown that for any $f$ and $\beta_{1}, \beta_{2}$ satisfying the assumptions of the lemma there exists a positive $\tilde{\eta}$ such that for all sufficiently large $\left|Z_{n}\right|$,

$$
\mathrm{E}_{Z_{n}}\left(\left|R_{n}\left(Z_{n}, \Delta_{n}, f, \beta, 2\right)\right|\right) \leqslant \tilde{c}\left|Z_{n}\right|^{2 \beta-2-\tilde{\eta}}\left|f^{\prime \prime} \circ \psi_{\beta_{1}, \beta_{2}}\left(Z_{n}\right)\right| .
$$

Although the function $f(x)=\exp (\kappa x)$ does not satisfy the conditions of Lemma 11 , it can be shown using arguments similar to those used in the proof of Lemma 11 that if $\beta=0$, then (61) still holds (we leave this to the reader).

From the moment conditions of the transition mechanism and the form of the mapping $\Psi$ it follows that on $\left\{Z_{n} \in G^{0}\right\}$,

$$
\begin{aligned}
& \mathrm{E}_{Z_{n}}\left(\nabla \psi_{\beta_{1}, \beta_{2}}\left(Z_{n}\right), \Delta_{n}\right)=0, \\
& \mathrm{E}_{Z_{n}}\left(\nabla \psi_{\beta_{1}, \beta_{2}}\left(Z_{n}\right), \Delta_{n}\right)^{2}=\left|\nabla \psi_{\beta_{1}, \beta_{2}}\left(Z_{n}\right)\right|^{2}=d^{2}(\beta)\left|Z_{n}\right|^{2 \beta-2} \text { and } \\
& \mathrm{E}_{Z_{n}}\left(D^{2} \psi_{\beta_{1}, \beta_{2}}\left(Z_{n}, \Delta_{n}, \Delta_{n}\right)\right)=\triangle \psi_{\beta_{1}, \beta_{2}}\left(Z_{n}\right)=0,
\end{aligned}
$$

where $d(\beta)$ is a positive constant (whose precise expression can be found in (24) of Aspandiiarov and Iasnogorodski (1997). Hence, (60) and (61) imply that there are positive constants $a_{0}, b_{0}, C$ such that for any $n \geqslant 0$ and for any $|z|>a_{0}, P_{z}$-a.s.,

$$
\mathrm{E}_{Z_{n}}\left(g\left(Z_{n+1}\right)-g\left(Z_{n}\right)\right) \geqslant C T^{\prime \prime}\left(\psi_{\alpha_{1}, \alpha_{2}}\right)\left(Z_{n}\right)\left|Z_{n}\right|^{2 \alpha-2} \geqslant 0, \quad \text { on }\left\{Z_{n} \in G^{0}\right\} \cap\left\{\left|Z_{n}\right|>A\right\} \text {. }
$$

Let us now prove that the conditional increments on the boundary $\partial G_{1} \cup \partial G_{2}$ can be made positive by an appropriate choice of $\kappa$. Observe that by formula (57) in Aspandiiarov and Iasnogorodski (1997) we have $\mathrm{E}_{Z_{n}}\left(\nabla \psi_{\alpha_{1}, \alpha_{2}}\left(Z_{n}\right), \Delta_{n}\right)=0$. On the other hand, easy calculations for the gradient and the second partial derivatives of the function $\psi_{\alpha_{1}, \alpha_{2}}$ show that for each $k=1,2$ on $\left\{Z_{n} \in \partial G_{k}\right\}$,

$$
\begin{aligned}
\mathrm{E}_{Z_{n}}\left(g^{\prime \prime}\left(Z_{n}\right)\left(\nabla \psi_{\alpha_{1}, \alpha_{2}}\left(Z_{n}\right), \Delta_{n}\right)^{2}+g^{\prime}\left(Z_{n}\right) D^{2} \psi_{\alpha_{1}, \alpha_{2}}\left(Z_{n}, \Delta_{n}, \Delta_{n}\right)\right) \\
=T^{\prime \prime}\left(\psi_{\alpha_{1}, \alpha_{2}}\right)\left(Z_{n}\right)\left|Z_{n}\right|^{2 \alpha-2}\left[c_{1}(\alpha) \mathrm{E}_{Z_{n}}\left(\left(\overrightarrow{\boldsymbol{v}}_{k}, \Delta_{n}\right)^{2}\right)+\frac{1}{c_{2}(\kappa)} h_{k}\left(\theta_{Z_{n}}\right)\right] \\
=T^{\prime \prime}\left(\psi_{\alpha_{1}, \alpha_{2}}\right)\left(Z_{n}\right)\left|Z_{n}\right|^{2 \alpha-2}\left[c_{1}(\alpha)\left(A_{k} \overrightarrow{\boldsymbol{v}}_{k}, \overrightarrow{\boldsymbol{v}}_{k}\right)+\frac{1}{c_{2}(\kappa)} h_{k}\left(\theta_{Z_{n}}\right)\right]
\end{aligned}
$$


where $\theta_{Z_{n}}$ is the angular coordinate of $Z_{n}, A_{k}$ are the covariance matrices of the transition mechanism from the boundaries $\partial G_{k}, \quad \vec{v}_{1}=\left(\cos \left(\alpha_{1}\right),-\sin \left(\alpha_{1}\right)\right), \quad \vec{v}_{2}=\left(\cos \left(\xi-\alpha_{2}\right)\right.$, $\left.\sin \left(\xi-\alpha_{2}\right)\right), h_{k}$ are some bounded functions and

$$
c_{1}(\alpha)=\left\{\begin{array}{ll}
\alpha^{2}, & \text { if } \alpha \neq 0, \\
\cos ^{-2}\left(\alpha_{1}\right), & \text { if } \alpha=0,
\end{array} \quad c_{2}(\kappa)= \begin{cases}\kappa-1, & \text { if } \alpha \neq 0 \\
\kappa, & \text { if } \alpha=0\end{cases}\right.
$$

Recall that by assumption $A_{k}, k=1,2$, are positive definite. Hence, for any $\kappa>1+\max \left(\left\|h_{k}\right\|\right) / \min \left(c_{1}(\alpha)\left(A_{k} \vec{v}_{k}, \vec{v}_{k}\right)\right)$ there exists a constant $c_{3}$ such that

$$
\begin{gathered}
\mathrm{E}_{Z_{n}}\left(g^{\prime \prime}\left(Z_{n}\right)\left(\nabla \psi_{\alpha_{1}, \alpha_{2}}\left(Z_{n}\right), \Delta_{n}\right)^{2}\right. \\
\left.+g^{\prime}\left(Z_{n}\right) D^{2} \psi_{\alpha_{1}, \alpha_{2}}\left(Z_{n}, \Delta_{n}, \Delta_{n}\right)\right) \\
\geqslant c_{3} T^{\prime \prime}\left(\psi_{\alpha_{1}, \alpha_{2}}\right)\left(Z_{n}\right)\left|Z_{n}\right|^{2 \alpha-2} .
\end{gathered}
$$

From (65) and (61) it can be seen that there exists a positive constant $c_{2}$ such that for all sufficiently large $a$ and for all $n$ on $\left\{Z_{n} \in \partial G_{k}\right\} \cap\left\{\left|Z_{n}\right|>a\right\}, k \in\{1,2\}$,

$$
\mathrm{E}_{Z_{n}}\left(g\left(Z_{n+1}\right)-g\left(Z_{n}\right)\right) \geqslant 0 .
$$

This and (63) together finish the proof of Lemma 4 for all sufficiently large $a$ and a certain number $\kappa$.

We now fix such $\kappa$. Let $z$ be any initial state from $F_{b} \backslash F_{b-K_{\alpha}}$. By the boundedness of jumps $P_{z}$-a.s., $b^{\kappa \alpha} \leqslant g\left(Z_{\tilde{\tau}_{b}}\right) \leqslant\left(b+K_{\alpha}\right)^{\kappa \alpha}$. Moreover, $\inf _{z \in F_{b} \backslash F_{b-K \alpha}} g(z) \geqslant\left(b-K_{\alpha}\right)^{\kappa \alpha}$. By assertion (ii) of Lemma 2, for all sufficiently large $b$ and any $z \in F_{b} \backslash F_{b-K_{a}}$,

$$
P_{z}\left(\tau_{a}<\tilde{\tau}_{b}\right) \leqslant \frac{\left(b+K_{\alpha}\right)^{\kappa \alpha}-\left(b-K_{\alpha}\right)^{\kappa \alpha}}{b^{\kappa \alpha}-a^{\kappa \alpha}} \leqslant \frac{c_{1}}{b},
$$

where the constant $c_{1}$ does not depend on $b$, and Proposition 2 is proved.

Let $\kappa$ and $\varepsilon$ be any positive numbers such that $\kappa<\min \left(1, \alpha^{-1}\right)$. Since $\alpha \geqslant 0$ and $\alpha_{i} \in(-\pi / 2, \pi / 2)$, there are fixed $\beta_{1}$ and $\beta_{2}$ such that $\beta_{i} \in(-\pi / 2, \pi / 2), \beta_{1}+\beta_{2}>0$ and $\alpha_{i}<\beta_{i}\left(\alpha_{i}>\beta_{i}\right)$ for $i=1,2$, if $\alpha>1$ or $\alpha=0 \quad(0<\alpha \leqslant 1)$. Set $\beta=\left(\beta_{1}+\beta_{2}\right) / \xi$ and $v=(\alpha-1) / \beta$. Define the function $g$ by $g(z)=T_{1}\left(\psi_{\alpha_{1}, \alpha_{2}}\right)(z)+T_{2}\left(\psi_{\beta_{1}, \beta_{2}}\right)(z)$, where

$$
\begin{aligned}
& T_{1}(x)= \begin{cases}x\left(1-x^{-\kappa}\right), & \text { if } \alpha \neq 0, \\
x\left(1-\log ^{-\kappa}(x)\right), & \text { if } \alpha=0,\end{cases} \\
& T_{2}(x)= \begin{cases}x^{\nu} \log ^{\varepsilon}(x), & \text { if } \alpha \neq 0, \alpha \neq 1, \\
\log ^{1+\varepsilon}(x), & \text { if } \alpha=1, \\
\left(1-x^{-1 / \beta}\right) \log (x), & \text { if } \alpha=0 .\end{cases}
\end{aligned}
$$

The following result will be proved in Appendix A.

Lemma 5. There exists $a_{0}>0$ such that for all $a \geqslant a_{0}$ and for any $z \in F_{a}^{c}$, the process $\left\{g\left(Z_{n \wedge \tau_{a_{0}}}\right), n \geqslant 0\right\}$ is a $P_{z}$-submartingale. 
Let $b$ be any fixed sufficiently large positive number greater than $\left(a_{0} \vee K_{\alpha}\right)\left(K_{\alpha}\right.$ is the constant from (47)). Set

$$
A=F_{a_{0}} ; \quad B=F_{b+K_{\alpha}} \backslash F_{b} .
$$

From the definition (47) of $K_{\alpha}$ and (41) it is immediate that

$$
\Gamma_{A, B} \subseteq F_{b} \backslash F_{b-K_{\alpha}}, \quad \Gamma_{A, B}^{\prime} \subseteq F_{b+K_{\alpha}}^{c}, \quad \Gamma_{A, B}^{\prime \prime} \subseteq F_{b}^{c} .
$$

As will now be shown, the function $g$ satisfies the conditions of Lemma 2 with $g, A$ and $D=\Gamma_{A, B}^{\prime \prime}$. This will then provide necessary bounds for the terms appearing in the bound of Theorem 3 and complete the proof of the desired lower bound.

The 'monotonicity' property of $\psi_{\alpha_{1}, \alpha_{2}}, \psi_{\beta_{1}, \beta_{2}}$ and the choice of $v, \beta_{1}$ and $\beta_{2}$ imply that whenever $Z_{0}=z \in(A \cup B)^{c}$,

$$
\bar{d}(b) \geqslant g\left(Z_{\tau_{\Gamma_{A, B}^{\prime \prime}}}\right) \geqslant \underline{d}(b) \text { and } \quad a \geqslant g\left(Z_{\tau_{A}}\right), \quad P_{z} \text {-a.s. }
$$

where $a=\sup _{z \in A} g(z)$, and the functions $\bar{d}(x), \underline{d}(x)$ are defined for all sufficiently large positive $x$ by $\bar{d}(x)=\bar{d}_{1}(x)+\bar{d}_{2}(x) ; \underline{d}(x)=\underline{d}_{1}(x)+\underline{d}_{2}(x)$, where $\underline{d}_{2}(x)=0$,

$$
\bar{d}_{1}(x)=\left\{\begin{array}{ll}
T_{1}\left(\left(x+K_{\alpha}\right)^{\alpha}\right), & \text { if } \alpha \neq 0, \\
T_{1}\left(\log \left(x+K_{\alpha}\right)\right), & \text { if } \alpha=0,
\end{array} \quad \underline{d}_{1}(x)= \begin{cases}T_{1}\left(x^{\alpha}\right), & \text { if } \alpha \neq 0, \\
T_{1}(\log x), & \text { if } \alpha=0,\end{cases}\right.
$$

and

$$
\bar{d}_{2}(x)= \begin{cases}(c(\beta))^{1-\alpha} x^{\alpha-1} \log ^{\varepsilon}\left(\left(c(\alpha)\left(x+K_{\alpha}\right)\right)^{\beta}\right), & \text { if } \alpha \in(0,1), \\ T_{2}\left(\left(c(\alpha)\left(x+K_{\alpha}\right)\right)^{\beta}\right), & \text { otherwise. }\end{cases}
$$

Plainly, for all sufficiently large $b, \underline{d}(b)>a$. Next, the irreducibility of the Markov chain $\left\{Z_{n}, n \geqslant 0\right\}$ implies that there exist $n>0, p_{0}>0, a_{1}>a_{0}, z^{\prime} \in \partial A$ and the state $y$ such that $\Psi_{\alpha_{1}, \alpha_{2}}\left(z^{\prime}\right) \leqslant a_{0}<\Psi_{\alpha_{1}, \alpha_{2}}(y)<a_{1}, g(y)>a+1$ and

$$
\left(A \cup F_{a_{1}}^{c}\right) P_{z^{\prime}, y}^{n}>p_{0} .
$$

Let us fix such $z^{\prime}, y$. By the first assertion of Lemma 2 and recalling the expression for $\bar{d}$, one easily sees that there exists $c_{1}>0$ such that for all large enough $b$,

$$
P_{z^{\prime}}\left(\tau_{A}>\tau_{\Gamma_{A, B}^{\prime \prime}}\right) \geqslant p_{0} P_{y}\left(\tau_{A}>\tau_{\Gamma_{A, B}^{\prime \prime}}\right) \geqslant p_{0} \frac{g(y)-a}{\bar{d}(b)} \geqslant \begin{cases}\frac{c_{1}}{b^{\alpha}}, & \text { if } \alpha \neq 0, \\ \frac{c_{1}}{\log (b)}, & \text { if } \alpha=0 .\end{cases}
$$

By Proposition 2 there exists a positive constant $c_{3}$ such that for all $b>b_{1}, \sup _{z \in \Gamma_{A, B}}$ $P_{z}\left(\tau_{A}<\tau_{\Gamma_{A, B}^{\prime \prime}}\right) \leqslant c_{3} / b$. The end of the proof is immediate. Let us fix any $b_{0}>\left(a_{1} \vee K_{\alpha} \vee b_{1}\right)$ such that for all $b>b_{0}$ inequality (75) holds. By the definition of $v_{B}, \mathrm{E}_{z}\left(v_{B}\right) \geqslant 1$ and $P_{z}\left(Z\left(v_{B}\right) \in \Gamma_{A, B}\right) \leqslant 1$, for any $z \in B$. We have thus estimated all the quantities appearing in (21) of Theorem 3 . The proof is concluded by substituting the bounds just obtained into (21) applied to sets $B$ from (69) with $b>b_{0}$. 


\subsection{Upper local bounds of Theorem 8}

As in the proof of the lower bounds, we start with a preliminary result which complements Lemma 2.

Proposition 3. Let $\alpha \geqslant 0$. There are positive constants $c, K \geqslant K_{\alpha}$ such that for all sufficiently large $a, b$ satisfying $b>a$ and for any $z \in F_{b-K} \backslash F_{b-2 K}, \quad P_{z}\left(\tau_{A}<\tilde{\tau}_{b}\right) \geqslant$ $c /[b \log (b)]$.

Proof. Fix any $\beta_{1}$ and $\beta_{2}$ such that $\beta_{i} \in(-\pi / 2, \pi / 2), \beta_{1}+\beta_{2}>0$ and $\alpha_{i}<\beta_{i}$. Let $\kappa$ be any positive number such that $\kappa<1$. Define the function $g$ by $g(z)=T_{1}\left(\psi_{\alpha_{1}, \alpha_{2}}\right)(z)+$ $T_{2}\left(\psi_{\beta_{1}, \beta_{2}}\right)(z)$, where $T_{2}(x)=c(\alpha, \beta) x^{-1 / \beta}$,

$$
T_{1}(x)= \begin{cases}\log (x), & \text { if } \alpha \neq 0, \\ x\left(1+x^{-\kappa}\right), & \text { if } \alpha=0,\end{cases}
$$

and $c(\alpha, \beta)$ is a positive constant to be chosen later.

Lemma 6. $\left\{g\left(Z_{n \wedge \tau_{a}}\right), n \geqslant 0\right\}$ is a $P_{z}$-supermartingale for all sufficiently large a.

Proof. Consider first the increments of $g\left(Z_{n}\right)$ when $Z_{n}$ belongs to the interior of the domain $G^{0}$. In this subcase Lemma 11 implies the existence of positive constants $a, c_{1}, c_{2}$ such that, for any $n \geqslant 0$ on $\left\{Z_{n} \in G^{0}\right\} \cap\left\{\left|Z_{n}\right|>a\right\}$,

$$
\begin{aligned}
& \mathrm{E}_{Z_{n}}\left(T_{1} \circ \psi_{\alpha_{1}, \alpha_{2}}\left(Z_{n+1}\right)-T_{1} \circ \psi_{\alpha_{1}, \alpha_{2}}\left(Z_{n}\right)\right) \leqslant-c_{1} f_{1}\left(Z_{n}\right), \\
& \left|\mathrm{E}_{Z_{n}}\left(T_{2} \circ \psi_{\beta_{1}, \beta_{2}}\left(Z_{n+1}\right)-T_{2} \circ \psi_{\beta_{1}, \beta_{2}}\left(Z_{n}\right)\right)\right| \leqslant c_{2} f_{2}\left(Z_{n}\right),
\end{aligned}
$$

where

$$
f_{1}(z)= \begin{cases}\psi_{\alpha_{1}, \alpha_{2}}^{-2}|z|^{2 \alpha-2}, & \text { if } \alpha \neq 0, \\ \psi_{\alpha_{1}, \alpha_{2}}^{-1-\kappa}|z|^{-2}, & \text { if } \alpha=0\end{cases}
$$

and $f_{2}(z)=c(\alpha, \beta) \psi_{\beta_{1}, \beta_{2}}^{-1 / \beta-2}(z)|z|^{2 \beta-2}$. By the 'monotonicity' property of the functions $\psi_{\alpha_{1}, \alpha_{2}}, \psi_{\beta_{1}, \beta_{2}}$, as $|z| \rightarrow \infty, f_{1}(z) / f_{2}(z) \rightarrow \infty$. Choosing sufficiently large $A$ and using again the 'monotonicity' property of $\psi_{\alpha_{1}, \alpha_{2}}$, it is seen that, for any $c(\alpha, \beta)$, there exist positive constants $c_{3}, a$ such that for all $n \geqslant 0$, on $\left\{Z_{n} \in G^{0}\right\} \cap\left\{\left|Z_{n}\right|>a\right\}$,

$$
\mathrm{E}_{Z_{n}}\left(g\left(Z_{n+1}\right)-g\left(Z_{n}\right)\right) \leqslant-c_{3} f_{1}\left(Z_{n}\right) \leqslant 0 .
$$

We can now handle the boundary subcase where we will have to choose the constant $c(\alpha, \beta)$. Again, Lemma 11 yields the existence of $a, c_{4}, c_{5}$ such that for all $n \geqslant 0$ on $\left\{Z_{n} \in \partial G_{i}\right\} \cap\left\{\left|Z_{n}\right|>a\right\}$ with $i=1,2$,

$$
\left\{\begin{array}{l}
\left|\mathrm{E}_{Z_{n}}\left(T_{1} \circ \psi_{\alpha_{1}, \alpha_{2}}\left(Z_{n+1}\right)-T_{1} \circ \psi_{\alpha_{1}, \alpha_{2}}\left(Z_{n}\right)\right)\right| \leqslant c_{4} f_{4}\left(Z_{n}\right), \\
\mathrm{E}_{Z_{n}}\left(T_{2} \circ \psi_{\beta_{1}, \beta_{2}}\left(Z_{n+1}\right)-T_{2} \circ \psi_{\beta_{1}, \beta_{2}}\left(Z_{n}\right)\right) \leqslant-c_{5} c(\alpha, \beta) f_{5}\left(Z_{n}\right),
\end{array}\right.
$$

where $f_{4}(z)=|z|^{-2}$ and $f_{5}(z)=\sin \left(\beta_{i}-\alpha_{i}\right) \psi_{\beta_{1}, \beta_{2}}^{-1 / \beta-1}(z)|z|^{\beta-1}$. By the 'monotonicity' property of $\psi_{\alpha_{1}, \alpha_{2}}, \psi_{\beta_{1}, \beta_{2}}$, there exists a positive constant $c_{6}$ such that as $|z| \rightarrow \infty, f_{4}(z) / f_{5}(z) \leqslant c_{6}$. 
Hence, choosing a sufficiently large $a$ and $c(\alpha, \beta)$ such that for all $n \geqslant 0$, on $\left\{Z_{n} \in \partial G_{i}\right\} \cap\left\{\left|Z_{n}\right|>a\right\}$

$$
\mathrm{E}_{Z_{n}}\left(g\left(Z_{n+1}\right)-g\left(Z_{n}\right)\right) \leqslant-c_{7} f_{5}\left(Z_{n}\right),
$$

for some constant $c_{7}$. The bounds (76) and (77) conclude the proof of the supermartingale property of the process $g(Z)$.

We are now ready to resume the proof of the proposition. Let $K$ be any positive number greater than $K_{\alpha}$ which is to be defined later. Also let $z$ be any arbitrary vector from $F_{b-K} \backslash F_{b-2 K}$. Set

$$
H(x)= \begin{cases}x^{\alpha}, & \text { if } \alpha \neq 0 \\ \log (x), & \text { if } \alpha=0\end{cases}
$$

Observe that $P_{z}$-a.s.,

$$
\begin{aligned}
& T_{1}(H(b)) \leqslant g\left(Z_{\tilde{\tau}_{b}}\right) \leqslant 2 T_{1}\left(H\left(b+K_{\alpha}\right)\right), \\
& \sup _{F_{b-K} \backslash F_{b-2 K}} g(z) \leqslant T_{1}(H(b-K))+\sup _{F_{b-K} \backslash F_{b-2 K}} T_{2}\left(\psi_{\beta_{1}, \beta_{2}}\right)(z) .
\end{aligned}
$$

Applying Lemma 1, we see that there exists a positive constant $K$ such that for all sufficiently large $b$ and any $z \in F_{b-K} \backslash F_{b-2 K}$,

$$
P_{z}\left(\tau_{A}<\tilde{\tau}_{b}\right) \geqslant \frac{T_{1}(H(b))-T_{1}(H(b-K))-\sup _{F_{b-K} \backslash F_{b-2 K}} T_{2}\left(\psi_{\beta_{1}, \beta_{2}}\right)(z)}{2 T_{1}\left(H\left(b+K_{\alpha}\right)\right)} \geqslant \frac{\frac{\alpha K}{b}-\frac{c_{1} c(\alpha, \beta)}{b-2 K}}{2 T_{1}\left(\left(b+K_{\alpha}\right)^{\alpha}\right)},
$$

where as usual $c_{1}$ does not depend on $b$. Choosing sufficiently large $K>c_{1} c(\alpha, \beta) / \alpha$ we obtain that there exists a positive constant $c_{2}$ such that for all sufficiently large $b$, $P_{z}\left(\tau_{A}<\tilde{\tau}_{b}\right) \geqslant c_{2} /[b \log (b)]$, as was to be proved.

Let us now fix any $K$ satisfying the conditions of Proposition 3. Let $\kappa$ and $\varepsilon$ be any positive numbers such that $\kappa<\min \left(1, \alpha^{-1}\right)$. Fix $\beta_{1}$ and $\beta_{2}$ such that $\beta_{i} \in(-\pi / 2, \pi / 2)$, $\beta_{1}+\beta_{2}>0$ and $\alpha_{i}>\beta_{i}, \quad\left(\alpha_{i}<\beta_{i}\right)$ for $i=1,2$, if $\alpha \geqslant 1, \alpha=0 \quad(0<\alpha<1)$. Set $\beta=\left(\beta_{1}+\beta_{2}\right) / \xi$ and $v=(\alpha-1) / \beta$. Let us define the function $g$ by $g(z)=$ $T_{1}\left(\psi_{\alpha_{1}, \alpha_{2}}\right)(z)+T_{2}\left(\psi_{\beta_{1}, \beta_{2}}\right)(z)$, where $T_{1}(x)=x\left(1+x^{-\kappa}\right)$ and

$$
T_{2}(x)= \begin{cases}x^{v} \log (x), & \text { if } \alpha \neq 0 \\ c(\alpha, \beta) x^{-1 / \beta}, & \text { if } \alpha=0\end{cases}
$$

where the constant $c(\alpha, \beta)$ is chosen as in Lemma 6. Again the main reason for the choice of $v, c(\alpha, \beta), \kappa, \beta_{1}, \beta_{2}$ is given by the following result.

Lemma 7. There exists $a_{0}>0$ such that for any $a \geqslant a_{0}$ and for any $z \in F_{a}^{c}$, the process $\left\{g\left(Z_{n \wedge \tau_{a_{0}}}\right), n \geqslant 0\right\}$ is a $\mathrm{P}_{\mathrm{z}}$-supermartingale.

The proof of this lemma is postponed until Appendix A.

Remark 18. It can be proved that in the case $\alpha>1$, the process $\left\{\log \left(\psi_{\alpha_{1}, \alpha_{2}}\left(Z_{n \wedge \tau_{a}}\right)\right), n \geqslant 0\right\}$ 
is a supermartingale for all sufficiently large a. Although in this case the existence of such simple supermartingale simplifies the consequent proof, it does not cover the remaining case $\alpha \leqslant 1$ and, therefore, we use the generic function defined in Lemma 7.

Let $a_{0}$ be any number satisfying the conditions of Lemma 7. For all sufficiently large $b>a_{0} \vee 2 K$, define the sets $A$ and $B$ by (69). For such sets $A, B$ property (70) holds. It will be now shown that the function $g$, the process $Z$ and the sets $A, D=\Gamma_{A, B}^{\prime \prime}$ satisfy the conditions of Lemma 1 for some initial values $z$.

The 'monotonicity' property of $\psi_{\alpha_{1}, \alpha_{2}}, \psi_{\beta_{1}, \beta_{2}}$ and the choice of $v, \beta_{1}$, and $\beta_{2}$ imply that (71) holds with the functions $\bar{d}(x)$ and $\underline{d}(x)$ defined, for all sufficiently large $x>0$, by

$$
\bar{d}(x)=\bar{d}_{1}(x)+\bar{d}_{2}(x), \quad \underline{d}(x)=\underline{d}_{1}(x)+\underline{d}_{2}(x),
$$

$\underline{d}_{2}(x)=0, \bar{d}_{1}, \underline{d}_{1}$ defined in (72) and

$$
\bar{d}_{2}(x)= \begin{cases}T_{2}(c(\alpha)(x+K)), & \text { if } \alpha \geqslant 1, \\ T_{2}(x / c(\beta)), & \text { if } \alpha<1\end{cases}
$$

Recall that $\Gamma_{A, B} \subseteq F_{b} \backslash F_{b-K}$ and $\bar{\Gamma}_{A, B}=F_{b-K} \backslash F_{b-2 K}$. Assertion (ii) of Lemma 3 yields that there exist positive constants $b_{1}, n_{1}, p_{1}$ independent of $b$ such that for all $b>b_{1}$ and for any $z \in \Gamma_{A, B}$ there exists $z^{\prime} \in \bar{\Gamma}_{A, B}$ satisfying ${ }_{\left(A \cup \Gamma_{A, B}^{\prime \prime}\right)} P_{z, z^{\prime}}^{n_{1}}>p_{1}$. Therefore,

$$
\inf _{z \in \Gamma_{A, B}} P_{z}\left(\tau_{A}<\tau_{\Gamma_{A, B}^{\prime \prime}}\right) \geqslant p_{1} \inf _{z \in \bar{\Gamma}_{A, B}} P_{z}\left(\tau_{A}<\tau_{\Gamma_{A, B}^{\prime \prime}}\right) .
$$

But by Proposition 3, there exists $c_{1}>0$ such that for all sufficiently large $b$,

$$
\inf _{z \in \bar{\Gamma}_{A, B}} P_{z}\left(\tau_{A}<\tau_{\Gamma_{A, B}^{\prime \prime}}\right) \geqslant \frac{c_{1}}{b \log (b)} .
$$

The Markov property and assertion (i) of Lemma 1 show that there exists $b_{2}>b_{1}$ such that, for all $b>b_{2}$ and for any $z \in \partial A$,

$$
P_{z}\left(\tau_{\Gamma_{A, B}^{\prime \prime}}<\tau_{A}\right)=\mathrm{E}_{z}\left(1_{\left(Z_{1} \in A^{c}\right)} P_{Z_{1}}\left(\tau_{\Gamma_{A, B}^{\prime \prime}}<\tau_{A}\right)\right) \leqslant \frac{\mathrm{E}_{z}\left(1_{\left(Z_{1} \in A^{c}\right)} g\left(Z_{1}\right)\right)}{\underline{d}(b)} .
$$

Recall that from the 'monotonicity' property of $\psi_{\alpha_{1}, \alpha_{2}}$ and the boundedness of the jumps of the Markov chain $Z$ it follows that there exists $c_{2}>0$ such that for any $z \in \partial A$, $\mathrm{E}_{z}\left(1_{\left(Z_{1} \in A^{c}\right)} g\left(Z_{1}\right)\right) \leqslant c_{2}$. Hence, there exists $c_{3}>0$ such that for all $b>b_{2}$ and for any $z \in \partial A$,

$$
P_{z}\left(\tau_{\Gamma_{A, B}^{\prime \prime}}<\tau_{A}\right) \leqslant \begin{cases}c_{3} b^{-\alpha}, & \text { if } \alpha \neq 0, \\ c_{3} \log (b), & \text { if } \alpha=0 .\end{cases}
$$

Obviously, $b_{2}$ can be chosen in such a way that for all $b>b_{2}$ inequality (84) holds. Finally, according to statement (ii) of Lemma 3 there exist $n_{2}, p_{2}, b_{3}$ such that for any $b>b_{3}$ and for any $z \in B, P_{z}\left(Z_{v_{B}} \in \Gamma_{A, B}, v_{B} \leqslant n_{2}\right) \geqslant p_{2}$ and, trivially, $\inf _{z \in B} P_{z}\left(Z_{v_{B}} \in \Gamma_{A, B}\right) \geqslant p_{2}$. Furthermore, it follows from the latter bounds, applying Remark 6, that for any $b>b_{3}$, $\sup _{z \in B} \mathrm{E}_{z}\left(v_{B}\right) \leqslant n_{2} / p_{2}$.

Applying Theorem 2 to the sets $A$ and $B$ with any $b>b_{1} \vee b_{2} \vee b_{3}$ and combining (7) with estimates $(83)-(86)$ concludes the proof of the local bounds. 


\subsection{Integrability results of Theorem 7}

Let $\mu, \zeta, k$ be any fixed positive numbers such that $\mu<\zeta$ and $k \geqslant 1$. Let $\beta_{i}, i=1,2$, be some fixed real numbers such that $\beta_{i} \in(-\pi / 2, \pi / 2)$ and $\beta_{i}<\alpha_{i}, \beta_{1}+\beta_{2}>0\left(\beta_{i}>\alpha_{i}\right)$ for $i=1,2$, in the case $\alpha \neq 0 \quad(\alpha=0)$. Set $\beta=\left(\beta_{1}+\beta_{2}\right) / \xi$ and $v=\alpha / \beta$. We define $g(z)=T_{1}\left(\psi_{\alpha_{1}, \alpha_{2}}\right)(z)+T_{2}\left(\psi_{\beta_{1}, \beta_{2}}\right)(z)$, where $T_{1}(x)=x \log _{k}^{-\mu}(x)$ and

$$
T_{2}(x)= \begin{cases}\frac{x^{v}}{\phi_{k}(\zeta, x)}, & \text { if } \alpha \neq 0 \\ \log _{k+1}^{-\zeta}(x), & \text { if } \alpha=0\end{cases}
$$

The proof of the next result is similar to that of Lemma 7 and is left to the reader.

Lemma 8. There exist positive constants $c_{1}$ and $a_{0}$ such that for all $a \geqslant a_{0}$ and for all $n \geqslant 0$, whenever $z \in F_{a}^{c}$,

$$
\mathrm{E}_{Z_{n}}\left(g\left(Z_{n+1}\right)-g\left(Z_{n}\right)\right) \leqslant-c_{1} f\left(Z_{n}\right), P_{z} \text {-a.s. on }\left\{\tau_{a}>n\right\},
$$

where the function $f$ is defined by

$$
f(z)= \begin{cases}|z|^{\alpha-2} / \phi_{k}(\mu,|z|), & \text { if } z \in G^{0}, \alpha \neq 0, \\ |z|^{\beta v-1} / \phi_{k}(\zeta,|z|)=|z|^{\alpha-1} / \phi_{k}(\zeta,|z|), & \text { if } z \in\left(\partial G_{1} \cup \partial G_{2}\right), \alpha \neq 0, \\ |z|^{-2} / \phi_{k+1}(\mu,|z|), & \text { if } z \in G^{0}, \alpha=0, \\ |z|^{-1} / \phi_{k+1}(\zeta,|z|), & \text { if } z \in\left(\partial G_{1} \cup \partial G_{2}\right), \alpha=0, \\ 0, & \text { if } z=(0,0) .\end{cases}
$$

The 'monotonicity' property of $\psi_{\alpha_{1}, \alpha_{2}}, \psi_{\beta_{1}, \beta_{2}}$ and the choice of $\zeta, \mu, \beta_{1}, \beta_{2}$ show that there exists $a_{1}>a_{0}$ such that $g(u)>f(u)$ if $u \in F_{a_{1}}^{c}$. The boundedness of jumps of the Markov chain $Z$ implies that for any $z \in F_{a_{1}}^{c}, \mathrm{E}_{z}\left(g\left(Z_{1}\right) 1_{\left(\tau_{a_{1}}>1\right)}\right)$ is finite. Remark 3 and (87) finish the verification of conditions of Theorem 1 with $A=F_{a_{1}}$, concluding the proof of the integrability results.

\subsection{Non-integrability results of Theorem 7}

We first prove the divergence results from (45). Suppose that the functions described in the theorem are integrable. Let $\mu$ be any fixed positive number, $k \geqslant 1$ and $\beta_{i}, i=1,2$, be some fixed real numbers such that $\beta_{i} \in(-\pi / 2, \pi / 2)$ and $\beta_{i}>\alpha_{i}$ for $i=1,2$. Set $\beta=\left(\beta_{1}+\beta_{2}\right) / \xi$. Let us define the function $g(z)$ by $g(z)=T\left(\psi_{\beta_{1}, \beta_{2}}\right)(z)$, where

$$
T(x)= \begin{cases}x^{\alpha / \beta} \log _{k}^{\mu}(x), & \text { if } \alpha \neq 0, \\ \log (x) \log _{k+1}^{\mu}(x), & \text { if } \alpha=0 .\end{cases}
$$

Lemma 9. There exist $a_{0}>0, c_{1}>0$ such that for all $a \geqslant a_{0}$ and for all $n \geqslant 0$, whenever $z \in F_{a}^{c}, P_{z}$-a.s.,

$$
\begin{cases}\mathrm{E}\left(g\left(Z_{n+1}\right)-g\left(Z_{n}\right) \mid \mathscr{F}_{n}\right) \leqslant 0, & \text { on }\left\{\tau_{F_{a}}>n\right\} \cap\left\{Z_{n} \in G^{0}\right\}, \\ \mathrm{E}\left(g\left(Z_{n+1}\right)-g\left(Z_{n}\right) \mid \mathscr{F}_{n}\right) \leqslant f\left(Z_{n}\right), & \text { on }\left\{\tau_{F_{a}}>n\right\} \cap\left\{Z_{n} \in \partial G\right\},\end{cases}
$$


where

$$
f(z)= \begin{cases}c_{1}|z|^{\alpha-1} \log _{k}^{\mu}(|z|), & \text { if } \alpha \neq 0 \\ c_{1}|z|^{-1} \log _{k+1}^{\mu}(|z|), & \text { if } \alpha=0\end{cases}
$$

Recall that the bound (75) implies the existence of $a_{1}>a_{0}, c_{2}>0, z \in G_{4}$ such that $\Psi_{\alpha_{1}, \alpha_{2}}(z) \in\left(a_{0}, a_{1}\right)$ and for all sufficiently large $b>a_{1}$,

$$
P_{z}\left(\tau_{a_{1}}>\tilde{\tau}_{b}\right) \geqslant \begin{cases}\frac{c_{2}}{b^{\alpha}}, & \text { if } \alpha \neq 0, \\ \frac{c_{2}}{\log (b)}, & \text { if } \alpha=0 .\end{cases}
$$

Set $A=F_{a_{1}}$ and fix any $z$ for which (90) holds. For all sufficiently large $b>a_{1}$, set $B=F_{b}^{c}$. Then, (89) easily implies that for all sufficiently large $b$,

$$
\begin{aligned}
\mathrm{E}_{z}\left(g\left(Z_{n+1 \wedge \tau_{A} \wedge \tau_{B}}\right)-g(z)\right) & \leqslant \sum_{k=0}^{n} \mathrm{E}_{z}\left(1_{\left(\tau_{A} \wedge \tau_{B}>k\right)} 1_{\left(Z_{k} \in \partial G_{1} \cup \partial G_{2}\right)} \mathrm{E}\left(g\left(Z_{k+1}\right)-g\left(Z_{k}\right) \mid \mathscr{F} k\right)\right) \\
& \leqslant \sum_{k=0}^{\infty} \mathrm{E}_{z}\left(1_{\left(\tau_{A} \wedge \tau_{B}>k\right)} 1_{\left(Z_{k} \in \partial G_{1} \cup \partial G_{2}\right)} f\left(Z_{k}\right)\right) \\
& \leqslant \sum_{k=0}^{\infty} \mathrm{E}_{z}\left(1_{\left(\tau_{A}>k\right)} 1_{\left(Z_{k} \in \partial G_{1} \cup \partial G_{2}\right)} f\left(Z_{k}\right)\right)
\end{aligned}
$$

Passing to the limit as $n \rightarrow \infty$ in the last estimate and using Fatou's lemma, we immediately obtain that

$$
\begin{aligned}
& \pi(z) \mathrm{E}_{z}\left(g\left(Z_{\tau_{B}}\right) 1_{\left(\tau_{A}>\tau_{B}\right)}-g(z)\right) \leqslant \pi(z) \mathrm{E}_{z}\left(g\left(Z_{\tau_{A} \wedge \tau_{B}}\right)-g(z)\right) \\
& \leqslant \sum_{k=0}^{\infty} \mathrm{E}_{z}\left(1_{\left(\tau_{A}>k\right)} 1_{\left(Z_{k} \in \partial G_{1} \cup \partial G_{2}\right)} f\left(Z_{k}\right)\right) .
\end{aligned}
$$

As above, the 'monotonicity' property of the functions $\psi_{\alpha_{1}, \alpha_{2}}, \psi_{\beta_{1}, \beta_{2}}$ implies that there exists $c_{3}>0$ such that $P_{z}$-a.s.,

$$
g\left(Z_{\tau_{B}}\right) \geqslant \begin{cases}c_{3} b^{\alpha} \log _{k}^{\mu}(b), & \text { if } \alpha>0, \\ c_{3} \log (b) \log _{k+1}^{\mu}(b), & \text { if } \alpha=0 .\end{cases}
$$

Putting this estimate and (90) into (92) and passing to the limit as $b \rightarrow \infty$,

$$
\sum_{k=0}^{\infty} \mathrm{E}_{z}\left(1_{\left(\tau_{A}>k\right)} 1_{\left(Z_{k} \in \partial G_{1} \cup \partial G_{2}\right)} f\left(Z_{k}\right)\right)
$$

should be infinite. By (5), the integral $\int \partial G_{1} \cup \partial G_{2} f(z) \pi(\mathrm{d} z)$ diverges and the desired contradiction follows. Hence $\int_{\partial \tilde{G}_{1} \cup \partial \tilde{G}_{2}} f(z) \tilde{\pi}(\mathrm{d} z)$ also diverges.

We now prove the divergence result in (44). To this end we will construct functions $f, g$, $h$ and a set $A$ satisfying the conditions of Remarks 3-4. Let us fix any positive numbers $\mu$, $\mu^{\prime}$, and $\xi$ such that $\mu>\mu^{\prime}$. Let $\beta_{1}, \beta_{2}$ be some fixed real numbers such that 
$\beta_{i} \in(-\pi / 2, \pi / 2)$ and $\beta_{i}>\alpha_{i}$, for $i=1,2$. Set $\beta=\left(\beta_{1}+\beta_{2}\right) / \xi$ and $v=\alpha / \beta$. Define $g(z)=T_{1}\left(\psi_{\alpha_{1}, \alpha_{2}}\right)(z)+T_{2}\left(\psi_{\beta_{1}, \beta_{2}}\right)(z)$ and $h(z)=T_{3}\left(\psi_{\alpha_{1}, \alpha_{2}}\right)(z)+T_{2}\left(\psi_{\beta_{1}, \beta_{2}}\right)(z)$, where $T_{1}(x)=$ $x \log _{k}^{\mu}(x), T_{3}(x)=x \log _{k}^{\mu^{\prime}}(x)$ and

$$
T_{2}(x)= \begin{cases}x^{v} \log ^{-1-\zeta}(x), & \text { if } \alpha \neq 0 \\ 1-\log _{k+1}^{-\zeta}(x), & \text { if } \alpha=0 .\end{cases}
$$

Observe that $g(z) / h(z) \rightarrow \infty$ as $|z| \rightarrow \infty$.

Lemma 10. There exist positive constants $a_{0}$ and $c_{1}$ such that for all $a \geqslant a_{0}$ and $n \geqslant 0$, whenever $z \in F_{a}^{c}, P_{z}$-a.s. on $\left\{\tau_{a}>n\right\}$, we have

$$
\left\{\begin{array}{l}
0 \leqslant \mathrm{E}_{Z_{n}}\left(g\left(Z_{n+1}\right)-g\left(Z_{n}\right)\right) \leqslant c_{1} f\left(Z_{n}\right), \\
0 \leqslant \mathrm{E}_{Z_{n}}\left(h\left(Z_{n+1}\right)-h\left(Z_{n}\right)\right),
\end{array}\right.
$$

where the function $f$ is defined by

$$
f(z)= \begin{cases}|z|^{\alpha-2} / \phi_{k}(-\mu,|z|), & \text { if } z \in G^{0}, \alpha \neq 0 ; \\ |z|^{\beta \nu-1} / \log ^{1+\zeta}(|z|)=|z|^{\alpha-1} / \log ^{1+\xi}(|z|), & \text { if } z \in\left(\partial G_{1} \cup \partial G_{2}\right), \alpha \neq 0 ; \\ |z|^{-2} / \phi_{k+1}(-\mu,|z|), & \text { if } z \in G^{0}, \alpha=0 ; \\ |z|^{-1} / \phi_{k+1}(\zeta,|z|), & \text { if } z \in\left(\partial G_{1} \cup \partial G_{2}\right), \alpha=0 ; \\ c, & \text { if } z=(0,0),\end{cases}
$$

with an arbitrary positive constant $c$. In particular, for any $a \geqslant a_{0}$ and for any $z \in F_{a}^{c}$ the processes $\left\{g\left(Z_{n \wedge \tau_{a}}\right), n \geqslant 0\right\},\left\{h\left(Z_{n \wedge \tau_{a}}\right), n \geqslant 0\right\}$ are $P_{z}$-submartingales.

The proof of the lemma is straightforward and is again left to the reader.

Let us now fix any $a \geqslant a_{0}$ and define $A=F_{a}$. Choose $a_{1}>a$ such that $\sup _{z \in A} h(z)<\inf _{z^{\prime} \in F_{a_{1}}^{c}} h\left(z^{\prime}\right)$ and fix any $z \in A, z^{\prime} \in F_{a_{1}}^{c}$ such that $F_{a_{1}} P_{z, z^{\prime}}^{n_{0}}>0$ for some $n_{0}$. Such $z, z^{\prime}, n_{0}$ exist by the irreducibility of $\left\{Z_{n}, n \geqslant 0\right\}$. The 'boundedness' of the jumps and the 'monotonicity' property of $\psi_{\alpha_{1}, \alpha_{2}}, \psi_{\beta_{1}, \beta_{2}}$ readily imply that for all $a$ and for all $z \in F_{a}, \mathrm{E}_{z}\left(g\left(Z_{1}\right) 1_{\left(\tau_{a}>1\right)}\right)$ and $\mathrm{E}_{z}\left(f\left(Z_{n \wedge \tau_{a}}\right)\right)$ are finite. Therefore, by Remark 4,

$$
\limsup _{n \rightarrow \infty}\left(g\left(Z_{n}\right) 1_{\left(\tau_{a}>n\right)}\right)=\infty \text {. }
$$

All conditions of Theorem 1' have now been verified. Hence $\int_{\tilde{G}} f(z) \tilde{\pi}(\mathrm{d} z)$ diverges. On the other hand, we have already shown in (45) that $\int_{\left(\partial \tilde{G}_{1} \cup \partial \tilde{G}_{2}\right)} f(z) \tilde{\pi}(\mathrm{d} z)$ converges. This immediately implies the desired divergence results in (44).

\subsection{Global bounds on the boundary}

The upper bounds in (49) and (50) easily follow from Proposition 1. To see this, recall that in Lemma 8 it was proved that there exist $a>0$, functions $f, g$ such that the set $A=F_{a}, f, g$ satisfy the conditions of Theorem 1 and $\mathrm{E}_{z}\left(g\left(Z_{1}\right) 1_{\left(\tau_{a}>1\right)}\right)$ is finite whenever $z \in F_{a}$. For all $b>a$, we now set $B=\left(F_{b} \backslash F_{a}\right) \cap\left(\partial G_{1} \cup \partial G_{2}\right)$ and $B=F_{b}^{c} \cap\left(\partial G_{1} \cup \partial G_{2}\right)$ in the respective cases $\alpha \leqslant 1$ and $\alpha>1$ and apply Proposition 1 . The asserted global upper bounds follow from the 'monotonicity' property of $\psi_{\alpha_{1}, \alpha_{2}}$. 
We now prove the global lower bounds. The case $\alpha=1$ is trivial, so suppose that $0<\alpha<1$. Let us first notice that since $0<\alpha<1$, and $\xi<\pi$, there exists a vector $v=\left(v_{1}, v_{2}\right)$ such that $v_{1}>0, v_{2}>0$, the function $g(z)=z \cdot v$ is non-negative on $G$ and $\max \left(\Phi\left(\mathrm{E}\left(Y_{n}^{(1)}\right)\right) \cdot v, \Phi\left(\mathrm{E}\left(Y_{n}^{(2)}\right)\right) \cdot v\right)>0$ (recall that the vectors $\Phi\left(\mathrm{E}\left(Y_{n}^{(1)}\right)\right), \Phi\left(\mathrm{E}\left(Y_{n}^{(2)}\right)\right)$ are the images of the vectors of the boundary reflection under the mapping $\Phi$ defined in Section 3.1). It can then be easily seen that (89) from the proof of the divergence results of Theorem 7 is valid with the function $f(z)=c_{1}$, where $c_{1}=\max \left(\Phi\left(\mathrm{E}\left(Y_{n}^{(1)}\right)\right) \cdot v\right.$, $\left.\Phi\left(\mathrm{E}\left(Y_{n}^{(2)}\right)\right) \cdot v\right)$ and $a_{0}=0$. This easily leads to (90) and (91) which, together with (3) and the 'monotonicity' property of $\psi_{\alpha_{1}, \alpha_{2}}$, imply that there exist $a_{1}, c_{2}, c_{3}, c_{4}>0$ such that for all $b>a_{1}$ and $z \in \partial F_{a_{1}}$,

$$
c_{2} \pi\left(c_{3} a_{1}<|z|<c_{4} b, z \in \partial G\right) \geqslant \pi(z) \mathrm{E}_{z}\left(g\left(Z_{\tau_{B}}\right) 1_{\left(\tau_{A}>\tau_{B}\right)}-g(z)\right) .
$$

The 'monotonicity' property of $\psi_{\alpha_{1}, \alpha_{2}}$ implies the existence of $c_{5}>0$ such that for all large $b, P_{z}$-a.s., $g\left(Z_{\tau_{B}}\right) \geqslant c_{5} b$. This, (90) and (94) concludes the proof of the lower bound in (50).

\subsection{Global bounds in the interior}

Lower and upper bounds in (51)-(52) in the case $\alpha \neq 0$ follow from (48), the upper bounds of Theorem 9 and 'monotonicity' property of $\psi_{\alpha_{1}, \alpha_{2}}$ by summation over $n$ of $\pi\left(F_{b+(n+1) K_{\alpha}} \backslash F_{b+n K_{\alpha}}\right)$ and $\pi\left(F_{a+(n+1) K_{\alpha}} \backslash F_{a+n K_{\alpha}}\right)$ in the respective cases $\alpha>2$ and $0<\alpha \leqslant 2$ and the 'monotonicity' property of $\psi_{\alpha_{1}, \alpha_{2}}$.

\section{Appendix A. Auxiliary sub- and super-martingale properties}

We now give the proof of Lemmas 5-9. All the proofs are based upon Lemma 2 from Aspandiiarov and Iasogonorodski (1997).

Definition 4. Let $\mathscr{G}$ be the following class of non-negative functions defined on $\mathbb{R}_{+}$:

$$
\begin{aligned}
\mathscr{G}= & \left\{T: \mathbb{R}_{+} \rightarrow \mathbb{R}_{+} ; T \in C^{3}(0, \infty),\right. \\
& \frac{T^{\prime \prime \prime}(x)}{T^{\prime \prime}(x)}=O\left(\frac{1}{x}\right) \text { and } \frac{T^{\prime \prime}(x)}{T^{\prime}(x)}=O\left(\frac{1}{x}\right) \text { as } x \rightarrow \infty, \\
& \forall v>0, \liminf _{x \rightarrow \infty}\left|\frac{T^{\prime \prime}(x) x^{1+v}}{T^{\prime}(x)}\right|>0 \text { and } \liminf _{x \rightarrow \infty}\left|\frac{T^{\prime}(x) x^{1+v}}{T(x)}\right|>0, \\
& \text { there exist positive } \left.a_{T}>1 \text { and } \tilde{A}_{T} \text { such that } \limsup _{x \rightarrow \infty} \frac{\left|T^{\prime \prime}\left(a_{T} x\right)\right|}{\left|T^{\prime \prime}(x)\right|} \leqslant \tilde{A}_{T}\right\} .
\end{aligned}
$$

The key to the proofs of the lemmas is the following result.

Lemma 11 (Aspandiiarov and Iasnogorodski 1997, Lemma 2). Let $\theta_{1}$ and $\theta_{2} \in(-\pi / 2, \pi / 2)$ be real numbers such that $\theta_{1}+\theta_{2} \geqslant 0$. Set $\theta=\left(\theta_{1}+\theta_{2}\right) / \xi$. Let $T$ be a 
function from $G$ such that in the case $\theta \neq 0 \quad(\theta=0)\left|T^{\prime \prime}\left(x^{\theta}\right)\right| x^{2 \theta-2} \quad\left(\left|T^{\prime \prime}(\log x)\right| x^{-2}\right)$ is monotone on some interval $[B, \infty)$. Suppose there exist positive constants $\chi \in(0,1)$ and $c$ such that for all $n \geqslant 0$ and for all $z, P_{z}$-a.s.,

$$
\begin{cases}\mathrm{E}_{Z_{n}}\left(\left|\Delta_{n}\right|^{2+\chi} \max \left(1,\left|T^{\prime \prime}\left(\left|\Delta_{n}\right|^{\theta}\right) \| \Delta_{n}\right|^{2 \theta-2}\right) \leqslant c,\right. & \text { if } \theta \neq 0 \\ \mathrm{E}_{Z_{n}}\left(\left|\Delta_{n}\right|^{2+\chi} \mid \max \left(1,\left|T^{\prime \prime}\left(|\log | \Delta_{n}||\right)\right|\left|\Delta_{n}\right|^{-2}\right) \leqslant c,\right. & \text { if } \theta=0\end{cases}
$$

where, as usual, we write for each $n \geqslant 0, \Delta_{n}=Z_{n+1}-Z_{n}$. Then there exist positive constants $A, b, C$ such that for any $n \geqslant 0$ and for any $|z|>A$ the following two statements hold $\mathrm{P}_{\mathrm{z}}$-a.s.:

(a) On $\left\{Z_{n} \in G^{0}\right\} \cap\left\{\left|Z_{n}\right|>A\right\}$,

$$
\left|\mathrm{E}_{Z_{n}}\left(T \circ \psi_{\theta_{1}, \theta_{2}}\left(Z_{n+1}\right)-T \circ \psi_{\theta_{1}, \theta_{2}}\left(Z_{n}\right)\right)\right| \leqslant b\left|T^{\prime \prime} \circ \psi_{\theta_{1}, \theta_{2}}\left(Z_{n}\right)\right|\left|Z_{n}\right|^{2 \theta-2} .
$$

Furthermore,

$$
\operatorname{sgn}\left(T^{\prime \prime} \circ \psi_{\theta_{1}, \theta_{2}}\left(Z_{n}\right)\right) \mathrm{E}_{Z_{n}}\left(T \circ \psi_{\theta_{1}, \theta_{2}}\left(Z_{n+1}\right)-T \circ \psi_{\theta_{1}, \theta_{2}}\left(Z_{n}\right)\right) \geqslant C\left|T^{\prime \prime} \circ \psi_{\theta_{1}, \theta_{2}}\left(Z_{n}\right)\right|\left|Z_{n}\right|^{2 \theta-2} .
$$

(b) For each $i=1,2$, we have on $\left\{Z_{n} \in \partial G_{i}\right\} \cap\left\{\left|Z_{n}\right|>A\right\}$,

$$
\left|\mathrm{E}_{Z_{n}}\left(T \circ \psi_{\theta_{1}, \theta_{2}}\left(Z_{n+1}\right)-T \circ \psi_{\theta_{1}, \theta_{2}}\left(Z_{n}\right)\right)\right| \leqslant \begin{cases}b\left|T^{\prime} \circ \psi_{\theta_{1}, \theta_{2}}\left(Z_{n}\right)\right|\left|Z_{n}\right|^{\theta-2}, & \text { if } \theta_{i}=\alpha_{i}, \\ b\left|T^{\prime} \circ \psi_{\theta_{1}, \theta_{2}}\left(Z_{n}\right) \| Z_{n}\right|^{\theta-1}, & \text { otherwise. }\end{cases}
$$

Furthermore,

$$
\begin{aligned}
\operatorname{sgn}\left(T^{\prime} \circ \psi_{\theta_{1}, \theta_{2}}\left(Z_{n}\right) \sin \left(\theta_{i}-\alpha_{i}\right)\right) \mathrm{E}_{Z_{n}}\left(T \circ \psi_{\theta_{1}, \theta_{2}}\left(Z_{n+1}\right)\right. & \left.-T \circ \psi_{\theta_{1}, \theta_{2}}\left(Z_{n}\right)\right) \\
& \geqslant C\left|T^{\prime} \circ \psi_{\theta_{1}, \theta_{2}}\left(Z_{n}\right) \sin \left(\theta_{i}-\alpha_{i}\right) \| Z_{n}\right|^{\theta-1} .
\end{aligned}
$$

Proof of Lemma 5. As is easy to see, the triplets $\left(T_{1}, \alpha_{1}, \alpha_{2}\right)$ and $\left(T_{2}, \beta_{1}, \beta_{2}\right)$ satisfy the conditions of Lemma 11. Let us now see consequences of this.

Consider first the increments of $g\left(Z_{n}\right)$ when $Z_{n}$ belongs to the interior of the domain $G^{0}$. In this subcase Lemma 11 implies that there exist positive constants $A, c_{1}, c_{2}$ such that, for any $n \geqslant 0$ on $\left\{Z_{n} \in G^{0}\right\} \cap\left\{\left|Z_{n}\right|>A\right\}$,

$$
\begin{aligned}
& \mathrm{E}_{Z_{n}}\left(T_{1} \circ \psi_{\alpha_{1}, \alpha_{2}}\left(Z_{n+1}\right)-T_{1} \circ \psi_{\alpha_{1}, \alpha_{2}}\left(Z_{n}\right)\right) \geqslant c_{1} f_{1}\left(Z_{n}\right), \\
& \left|\mathrm{E}_{Z_{n}}\left(T_{2} \circ \psi_{\beta_{1}, \beta_{2}}\left(Z_{n+1}\right)-T_{2} \circ \psi_{\beta_{1}, \beta_{2}}\left(Z_{n}\right)\right)\right| \leqslant c_{2} f_{2}\left(Z_{n}\right),
\end{aligned}
$$

where

$$
\begin{gathered}
f_{1}(z)= \begin{cases}\left(\kappa-\kappa^{2}\right) \psi_{\alpha_{1}, \alpha_{2}}(z)^{-(\kappa+1)} \psi_{\alpha_{1}, \alpha_{2}}(z)|z|^{2 \alpha-2}, & \text { if } \alpha \neq 0 ; \\
\log ^{-\mu-1}\left(\psi_{\alpha_{1}, \alpha_{2}}(z)\right) \psi_{\alpha_{1}, \alpha_{2}}^{-1}(z)|z|^{-2}, & \text { if } \alpha=0 .\end{cases} \\
f_{2}(z)= \begin{cases}\psi_{\beta_{1}, \beta_{2}}^{\nu-2}(z) \log ^{\mathrm{e}}\left(\psi_{\beta_{1}, \beta_{2}}(z)\right)|z|^{2 \beta-2}, & \text { if } \alpha \neq 0 \\
\psi_{\beta_{1}, \beta_{2}}^{-2-1 / \beta}(z)|z|^{2 \beta-2} \log \left(\psi_{\beta_{1}, \beta_{2}}^{-1-1 / \beta}(z)\right), & \text { if } \alpha=0 .\end{cases}
\end{gathered}
$$

But, by the choice of $\kappa, v$ and the 'monotonicity' property of the functions $\psi_{\alpha_{1}, \alpha_{2}}, \psi_{\beta_{1}, \beta_{2}}$ we know that as $|z| \rightarrow \infty, f_{1}(z) / f_{2}(z) \rightarrow \infty$. Choosing sufficiently large $A$ and again using the 
'monotonicity' property of $\psi_{\alpha_{1}, \alpha_{2}}$, we see that there exists a positive constant $c_{3}$ such that for all $n \geqslant 0$, on $\left\{Z_{n} \in G^{0}\right\} \cap\left\{\left|Z_{n}\right|>A\right\}$,

$$
\mathrm{E}_{Z_{n}}\left(g\left(Z_{n+1}\right)-g\left(Z_{n}\right)\right) \geqslant c_{3} f_{1}\left(Z_{n}\right)
$$

We can now handle the boundary subcase. Again, Lemma 11 yields the existence of $A, c_{4}, c_{5}$ such that, for all $n \geqslant 0$ on $\left\{Z_{n} \in \partial G_{i}\right\} \cap\left\{\left|Z_{n}\right|>A\right\}, i=1,2$,

$$
\left\{\begin{array}{l}
\left|\mathrm{E}_{Z_{n}}\left(T_{1} \circ \psi_{\alpha_{1}, \alpha_{2}}\left(Z_{n+1}\right)-T_{1} \circ \psi_{\alpha_{1}, \alpha_{2}}\left(Z_{n}\right)\right)\right| \leqslant c_{4} f_{4}\left(Z_{n}\right), \\
\mathrm{E}_{Z_{n}}\left(T_{2} \circ \psi_{\beta_{1}, \beta_{2}}\left(Z_{n+1}\right)-T_{2} \circ \psi_{\beta_{1}, \beta_{2}}\left(Z_{n}\right)\right) \geqslant c_{5} f_{5}\left(Z_{n}\right),
\end{array}\right.
$$

where $f_{4}(z)=|z|^{\alpha-2}$ and

$$
f_{5}(z)= \begin{cases}v \sin \left(\beta_{i}-\alpha_{i}\right) \psi_{\beta_{1}, \beta_{2}}^{v-1}(z) \log ^{\varepsilon}\left(\psi_{\beta_{1}, \beta_{2}}(z)\right)|z|^{\beta-1}, & \text { if } \alpha \neq 0, \alpha \neq 1 ; \\ \sin \left(\beta_{i}-\alpha_{i}\right) \log ^{\varepsilon}\left(\psi_{\beta_{1}, \beta_{2}}(z)\right)|z|^{\beta-1}, & \text { if } \alpha=1 ; \\ \sin \left(\beta_{i}-\alpha_{i}\right) \psi_{\beta_{1}, \beta_{2}}^{-1-1 / \beta}(z)|z|^{\beta-1} \log \left(\psi_{\beta_{1}, \beta_{2}}^{-1-1 / \beta}(z)\right), & \text { if } \alpha=0 .\end{cases}
$$

Again the choice of $v, \beta_{1}, \beta_{2}$ and the 'monotonicity' property of $\psi_{\alpha_{1}, \alpha_{2}}, \psi_{\beta_{1}, \beta_{2}}$ imply that as $|z| \rightarrow \infty, f_{4}(z) / f_{5}(z) \rightarrow 0$. Hence, for a sufficiently large $A$ and a positive constant $c_{6}$, for all $n \geqslant 0$, on $\left\{Z_{n} \in \partial G_{i}\right\} \cap\left\{\left|Z_{n}\right|>A\right\}$,

$$
\mathrm{E}_{Z_{n}}\left(g\left(Z_{n+1}\right)-g\left(Z_{n}\right)\right) \geqslant c_{6} f_{5}\left(Z_{n}\right)
$$

Inequalities (100) and (101) conclude the proof.

Proof of Lemma 7. The case $\alpha=0$ has already been considered, so that the case $\alpha>0$ is all that is left. Again we separate two subcases.

(i) Interior $G^{0}$. Lemma 11 ensures that there exist positive constants $A, c_{1}, c_{2}$ such that, for any $n \geqslant 0$ on $\left\{Z_{n} \in G^{0}\right\} \cap\left\{\left|Z_{n}\right|>A\right\}$,

$$
\begin{aligned}
& \mathrm{E}_{Z_{n}}\left(T_{1} \circ \psi_{\alpha_{1}, \alpha_{2}}\left(Z_{n+1}\right)-T_{1} \circ \psi_{\alpha_{1}, \alpha_{2}}\left(Z_{n}\right)\right) \leqslant-c_{1} f_{1}\left(Z_{n}\right), \\
& \left|\mathrm{E}_{Z_{n}}\left(T_{2} \circ \psi_{\beta_{1}, \beta_{2}}\left(Z_{n+1}\right)-T_{2} \circ \psi_{\beta_{1}, \beta_{2}}\left(Z_{n}\right)\right)\right| \leqslant c_{2} f_{2}\left(Z_{n}\right),
\end{aligned}
$$

where $f_{1}(z)=\left(\kappa-\kappa^{2}\right) \psi_{\alpha_{1}, \alpha_{2}}^{-1-\kappa}(z)\left|Z_{n}\right|^{2 \alpha-2}$ and

$$
f_{2}(z)= \begin{cases}\psi_{\beta_{1}, \beta_{2}}^{v-2}(z) \log \left(\psi_{\beta_{1}, \beta_{2}}(z)\right)|z|^{2 \beta-2}, & \text { if } \alpha \neq 0, \neq 1, \\ |z|^{2 \beta-2} / \psi_{\beta_{1}, \beta_{2}}^{2}(z), & \text { if } \alpha=1 .\end{cases}
$$

The choice of $\kappa$ and and the 'monotonicity' property of the functions $\psi_{\alpha_{1}, \alpha_{2}}, \psi_{\beta_{1}, \beta_{2}}$ ensure that as $|z| \rightarrow \infty, f_{1}(z) / f_{2}(z) \rightarrow \infty$. Recalling the 'monotonicity' property of $\psi_{\alpha_{1}, \alpha_{2}}$ and choosing sufficiently large $A$ and a positive constant $c_{3}$, it follows that for all $n \geqslant 0$, on $\left\{Z_{n} \in G^{0}\right\} \cap\left\{\left|Z_{n}\right|>A\right\}$,

$$
\mathrm{E}_{Z_{n}}\left(g\left(Z_{n+1}\right)-g\left(Z_{n}\right)\right) \leqslant-c_{3} f_{1}\left(\left|Z_{n}\right|\right) .
$$

(ii) In the remaining case, when the process jumps from the boundary $\partial G_{1} \cap \partial G_{2}$, Lemma 11 ensures the existence of $A, c_{4}, c_{5}$ such that, for all $n \geqslant 0$ on $\left\{Z_{n} \in \partial G_{i}\right\} \cap\left\{\left|Z_{n}\right|>A\right\}, i=1,2$, 


$$
\left\{\begin{array}{l}
\mathrm{E}_{Z_{n}}\left(T_{1} \circ \psi_{\alpha_{1}, \alpha_{2}}\left(Z_{n+1}\right)-T_{1} \circ \psi_{\alpha_{1}, \alpha_{2}}\left(Z_{n}\right)\right) \mid \leqslant c_{4} f_{3}\left(Z_{n}\right), \\
\mathrm{E}_{Z_{n}}\left(T_{2} \circ \psi_{\beta_{1}, \beta_{2}}\left(Z_{n+1}\right)-T_{2} \circ \psi_{\beta_{1}, \beta_{2}}\left(Z_{n}\right)\right) \leqslant-c_{5} v \sin \left(\alpha_{i}-\beta_{i}\right) f_{4}\left(Z_{n}\right),
\end{array}\right.
$$

where $f_{3}(z)=\psi_{\alpha_{1}, \alpha_{2}}(z)|z|^{-2}$ and

$$
f_{4}(z)= \begin{cases}\psi_{\beta_{1}, \beta_{2}}^{v-1}(z) \log \left(\psi_{\beta_{1}, \beta_{2}}(z)\right)|z|^{\beta-1}, & \text { if } \alpha \neq 0, \neq 1, \\ |z|^{\beta-1} / \psi_{\beta_{1}, \beta_{2}}(z), & \text { if } \alpha=1 .\end{cases}
$$

In this case $f_{4}(z) / f_{3}(z) \rightarrow \infty$ as $|z| \rightarrow \infty$. Hence, there exists a positive constant $c_{6}$ such that for all sufficiently large $\left|Z_{n}\right|, \mathrm{E}_{Z_{n}}\left(g\left(Z_{n+1}\right)-g\left(Z_{n}\right)\right) \leqslant-c_{6} f_{4}\left(Z_{n}\right)$ which concludes the proof.

\section{Appendix B. Proof of auxiliary results on the geometry of the two-dimensional reflected random walks}

We start with one useful consequence of the moment condition in the interior of $G_{4}$.

Lemma 12. (i) For any straight line $L$ and for any $z \in G^{0} \cap L$, there exist at least two onestep transitions from $z$ to $z_{1}$ and $z_{2}$, where $z_{1}$ and $z_{2}$ belong to two open half-spaces separated by $L$.

(ii) For any $a \in \mathbb{R}^{2}$ and for any $\delta \in(0,2 \pi)$ there exist $a^{\prime} \in \mathbb{R}^{2}, n_{1}>0, b_{1}>0, M>0$ and $p_{1}>0$ such that $\left|\theta_{a}-\theta_{a^{\prime}}\right|<\delta$ and for any $z$ satisfying $|z|>b_{1}$ and $\operatorname{dist}(z, \partial G)>M$ we have $P_{z}\left(Z_{n_{1}}=z+a^{\prime}\right)=p_{1}$ and $P_{z}$-a.s., $\max _{k=1, n_{1}}\left|Z_{k}-z\right| \leqslant M$.

Proof. (i) The assertion is an immediate consequence of the mean-zero drifts condition $\mathrm{E}\left(Y^{(0)}\right)=(0,0)$ and the positive definiteness of the covariance matrix $A^{0}$.

(ii) The proof is almost immediate. It suffices to observe that zero drifts, the positive definiteness of the covariance matrix $A^{0}$ and the assertion of the first part of the lemma imply that there exist at least three directions of one-step transitions $a_{1}, a_{2}, a_{3}$ such that $\mathbb{R}^{2}$ is generated by their linear combinations with positive coefficients. Moreover, these transitions do not depend on $z$ because of the homogeneity of increments distributions in $G^{0}$.

We need another auxiliary result whose proof is easy and is omitted.

Lemma 13. Let $\alpha$ and $b$ be any positive constants. Let $f_{\alpha, b}$ be the curve defined by $\Psi_{\alpha_{1}, \alpha_{2}}(z)=b$, with $z \in G$. Let us also define the set $K=\{\theta \in(0, \xi)$; $\left.\sin \left((\alpha-1) \theta-\alpha_{1}\right)=0\right\}$. Then, only the following situations are possible:

(i) $K$ is empty and $f_{\alpha, b}$ is concave in $G$.

(ii) $K$ is empty and $f_{\alpha, b}$ is convex in $G$.

(iii) There exists a unique $\underline{\theta} \in K$ such that either $f_{\alpha, b}$ is concave in $G \cap\{\theta \in[0, \underline{\theta})\}$ and is convex in $G \cap\{\theta \in(\underline{\theta}, \xi]\}$ or $f_{\alpha, b}$ is convex in $G \cap\{\theta \in[0, \underline{\theta})\}$ and is concave in $G \cap\{\theta \in(\underline{\theta}, \xi]\}$. 
Proof of Lemma 3. Let us first study the increments of $\Psi_{\alpha_{1}, \alpha_{2}}$. Let $a$ be any fixed vector from $\mathbb{R}^{2}$. For each integer $n$ we will let

$$
\Delta_{a, n}\left(\Psi_{\alpha_{1}, \alpha_{2}}(z)\right)=\Psi_{\alpha_{1}, \alpha_{2}}(z+n a)-\Psi_{\alpha_{1}, \alpha_{2}}(z) .
$$

Plain calculations and the 'monotonicity' property of $\psi_{\alpha_{1}, \alpha_{2}}$ show that for any $a \in \mathbb{R}^{2}$ there exist positive constants $c_{2}, c_{3}$ such that as $|z| \rightarrow \infty$,

$$
\begin{aligned}
c_{2} n|a| \cos \left((\alpha-1) \theta_{z}-\alpha_{1}+\theta_{a}\right)+o(1) & \leqslant \Delta_{a, n}\left(\Psi_{\alpha_{1}, \alpha_{2}}(z)\right) \\
& \leqslant c_{3} n|a| \cos \left((\alpha-1) \theta_{z}-\alpha_{1}+\theta_{a}\right)+o(1)
\end{aligned}
$$

(here $o(1) \rightarrow 0$ as $|z| \rightarrow \infty$, uniformly on $a$ ).

(i) Let us now fix any vector $a^{\prime}$ such that $\theta_{a^{\prime}} \in\left[0,\left(\pi / 2-\alpha_{2}+\xi\right)\right)$ in the case $\alpha \geqslant 1$ and $\theta_{a^{\prime}} \in\left[\left(-\pi / 2-\alpha_{2}+\xi\right)^{+}, \xi \wedge\left(\alpha_{1}+\pi / 2\right)\right)$ in the case $\alpha<1$. The reason for this choice of $a^{\prime}$ lies in the fact that

$$
\min _{\theta \in[0, \xi]} \cos \left((\alpha-1) \theta-\alpha_{1}+\theta_{a^{\prime}}\right)>0
$$

Obviously, we can also suppose that for this vector $a^{\prime}$ Lemma 12 is applicable with some positive constants $M, b_{1}, n_{1}, p_{1}$. Let us now fix them. Next, easy geometrical arguments based on statement (i) of Lemma 12, on Lemma 13, and on the non-degeneracy condition of the boundary reflection show that there exist positive constants $b_{2}, n_{2}, p_{2}$ such that for all $b \geqslant b_{2}$, whenever $z \in \Gamma_{F_{a_{0}}, F_{b}}$, we have

$$
P_{z}\left(\operatorname{dist}\left(Z_{n_{2}}, f_{\alpha, b}\right)>M, \operatorname{dist}\left(Z_{n_{2}}, \partial G\right)>M, \tau_{F_{b, \alpha_{1}, \alpha_{2}, \xi}}>n_{2}\right) \geqslant p_{2} .
$$

Making $n_{2}$-step transitions away from the boundary and from the curve $f_{\alpha, b}$ and then $n_{1}$-step transitions along $a^{\prime}$ we get the desired assertion from the choice of $a^{\prime}$, (103) and (104).

(ii) The proof of the second statement needs more care, but the idea is basically the same and consists in using (103). Let $a_{1}$ be a vector such that

$$
\theta_{a_{1}} \in \begin{cases}\left(\pi+\xi, \frac{3 \pi}{2}+\alpha_{1}\right), & \text { if } \alpha<1 \text { and } \xi<\alpha_{1}+\frac{\pi}{2}, \\ \left(\pi+\xi,\left(\frac{3 \pi}{2}-\alpha_{2}+\xi\right) \wedge 2 \pi\right), & \text { otherwise. }\end{cases}
$$

Set also

$$
\bar{\theta}_{1}= \begin{cases}\frac{-\frac{3 \pi}{2}-\alpha_{1}+\theta_{a_{1}}}{1-\alpha}, & \begin{array}{l}
\text { if } \alpha<1 \text { and } \xi \geqslant \alpha_{1}+\frac{\pi}{2} \\
0,
\end{array} \text { otherwise. }\end{cases}
$$

(ii) Similarly, fix another vector $a_{2}$ such that 


$$
\theta_{a_{2}} \in \begin{cases}\left(\frac{\pi}{2}+\xi-\alpha_{2}, \pi\right), & \text { if } \alpha<1 \text { and } \xi<\alpha_{2}+\frac{\pi}{2}, \\ \left(\frac{\pi}{2}+\alpha_{1}, \pi\right), & \text { otherwise, }\end{cases}
$$

and $\theta_{a_{2}}>\pi+\theta_{a_{1}}$ if $\xi \geqslant\left(\alpha_{1} \vee \alpha_{2}\right)+\frac{\pi}{2}$. Set also

$$
\bar{\theta}_{2}= \begin{cases}\frac{-\frac{\pi}{2}-\alpha_{1}+\theta_{a_{1}}}{1-\alpha}, & \begin{array}{l}
\text { if } \alpha<1 \text { and } \xi \geqslant \alpha_{2}+\frac{\pi}{2}, \\
\pi,
\end{array} \text { otherwise. }\end{cases}
$$

Notice that the choice of $a_{1}, a_{2}$ ensures that $\bar{\theta}_{1}, \bar{\theta}_{2} \in[0, \xi], \bar{\theta}_{1}<\bar{\theta}_{2}$,

$$
\left\{\begin{array}{l}
\max \max _{\theta \in}\left[\frac{\bar{\theta}_{1}+\bar{\theta}_{2}}{2}, \xi\right]^{\cos \left((\alpha-1) \theta-\alpha_{1}+\theta_{a_{1}}\right)<0,} \\
\operatorname{ma}\left[0, \frac{\bar{\theta}_{1}+\bar{\theta}_{2}}{2}\right]^{\cos \left((\alpha-1) \theta-\alpha_{1}+\theta_{a_{2}}\right)<0 .}
\end{array}\right.
$$

As above, we can assume that the vectors $a_{1}, a_{2}$ satisfy the conditions of Lemma 12 . Let us fix corresponding constants $p_{1}, n_{1}, b_{1}, M_{1}$ and $p_{2}, n_{2}, b_{2}, M_{2}$. We set $M=M_{1} \vee M_{2}$.

Next, easy geometrical arguments based on statement (i) of Lemma 12, on Lemma 13, and on the non-degeneracy condition of the boundary reflection show that there exist positive constants $b_{3}, n_{3}, p_{3}$ such that for all $b \geqslant b_{3}$, whenever $z \in \Gamma_{F_{a_{0}}, F_{b}}$, we have

$$
P_{z}\left(\operatorname{dist}\left(Z_{n_{3}}, f_{\alpha, b}\right)>M, \operatorname{dist}\left(Z_{n_{3}}, \partial G\right)>M, \tau_{F_{b, \alpha_{1}, \alpha_{2}, \xi}}>n_{3}\right) \geqslant p_{3} .
$$

Let us now take any $z \in \Gamma_{F_{a_{0}}, F_{b}}$. Making $n_{3}$-step transitions in such a way that they satisfy (107) and then moving along the vector $a_{1}\left(a_{2}\right)$, if $\theta_{z} \in\left[\left(\bar{\theta}_{1}+\bar{\theta}_{2}\right) / 2, \xi\right]$ $\left(\theta_{z} \in\left[0,\left(\bar{\theta}_{1}+\bar{\theta}_{2}\right) / 2\right]\right)$ we see from the left-hand side of (103) that in a finite time depending only on the vectors $a_{1}, a_{2}$ and $K$ we reach $F_{b-K}$, as asserted.

\section{References}

Asmussen, S. (1987) Applied Probability and Queues. Chichester: Wiley.

Aspandiiarov, S (1994) Some properties of Brownian motion and reflected Markov chains. Doctoral thesis. Université Paris VI, Paris.

Aspandiiarov, S. and Iasnogorodski, R. (1995) General results on the stationary distributions for countable Markov chains and their applications. Prepublication no. 273 of the Laboratoire de Probabilités, Université Paris VI.

Aspandiiarov, S. and Iasnogorodski, R. (1997) Tails of passage-time for non-negative stochastic processes and an application to stochastic processes with boundary reflection in a wedge. Stochastic Process. Appl., 66, 115-145.

Aspandiiarov, S. and Iasnogorodski, R. (1998) General criteria of integrability of functions of passagetimes for non-negative stochastic processes and their applications. Theory Probab. Appl., 43, $509-539$.

Aspandiiarov, S., Iasnogorodski, R. and Menshikov, M, (1996) Passage-time moments for non-negative 
stochastic processes and an application to reflected random walks in a quadrant. Ann. Probab., 24, 932-960.

Borovkov, A.A., Fayolle, G. and Korshunov, D.A. (1992) Transient phenomena for Markov chains and their applications. J. Appl. Probab., 24, 322-342.

Chung, K.L. (1967) Markov Chains with Stationary Transition Probabilities. Berlin: Springer-Verlag.

Fayolle, G., Malyshev, V. and Menshikov. M. (1992) Random walks in a quarter plane with zero drifts. Ann. Inst. H. Poincaré Probab. Statist., 28, 179-195.

Fayolle, G., Malyshev, V. and Menshikov. M. (1994) Topics in the Constructive Theory of Countable Markov Chains. Cambridge: Cambridge University Press.

Foster, F.G. (1953) On stochastic matrices associated with certain queueing processes. Ann. Math. Statist., 24, 355-360.

Lamperti, J. (1963) Criteria for stochastic processes II: Passage-time moments. J. Math. Anal. Appl., 7, $127-145$.

Menshikov, M. and Popov, S. (1995) Exact power estimates for countable Markov chains. Markov Process. Related Fields, 1, 57-78.

Meyn, S.P. and Tweedie, R.L. (1993) Markov Chains and Stochastic Stability. Berlin: Springer-Verlag.

Nummelin, E. and Tuominnen, P. (1982) Geometric ergodicity of Harris recurrent Markov chains with applications to renewal theory. Stochastic Process. Appl., 12, 187-202.

Nummelin, E. and Tweedie, R.L. (1978) Geometric ergodicity and R-positivity for general Markov chains. Ann. Probab., 6, 404-420.

Nummelin, E. and Tweedie, R.L. (1994) Subgeometric rates of convergence of f-ergodic Markov chains. Adv. Appl. Probab., 26, 775-798.

Tweedie, R.L. (1983) The existence of moments for stationary Markov chains. J. Appl. Probab., 28, 191-196.

Williams, R.J. (1985) Recurrence classification for reflected Brownian motion in a wedge, Ann. Probab., 13, 758-778.

Received November 1995 and revised October 1998. 